\title{
Modélisation et tests des effets d'une stratégie d'enrichissement expérientiel : application au contexte touristique
}

\begin{tabular}{|c|c|c|}
\hline Lydie Bonnefoy-Claudet & Rémi Mencarelli & Cindy Lombart \\
Maître de Conférences & Professeur des Universités & Professeur \\
IAE Savoie Mont Blanc - & IAE Savoie Mont Blanc - & AUDENCIA Nantes (PRES \\
Université de Savoie & Université de Savoie & LUNAM) \\
IREGE & IREGE & Centre de Recherche IN SITU \\
Adresse professionnelle : & Adresse professionnelle : & Adresse professionnelle : \\
IAE de Savoie Mont-Blanc - & IAE de Savoie Mont-Blanc - & AUDENCIA Nantes, Ecole de \\
IREGE Université de Savoie & IREGE Université de Savoie & Management \\
4, chemin de Bellevue & 4 chemin de Bellevue & 8 route de la Jonelière \\
BP 80439 & BP 80439 & BP 31222 \\
74944 Annecy-le-vieux Cedex & 74944 Annecy-le-vieux Cedex & 44312 Nantes Cedex 3 \\
Tel : 04 50 09 24 74 & Tel : 04 50 09 24 00 & Tel : 02 40 37 34 40 \\
Fax : 04 50 09 24 39 & Fax : 04 50 09 24 10 & Fax : 02 40 37 34 07 \\
E-Mail : lydie.bonnefoy- & remimencarelli@yahoo.fr & E-Mail : clombart@audencia.com \\
claudet@univ-savoie.fr & & \\
\hline
\end{tabular}

Remerciements : Les auteurs remercient les évaluateurs et le rédacteur en chef dont les remarques ont permis d'améliorer significativement ce papier. 


\title{
Modélisation et tests des effets d'une stratégie d'enrichissement expérientiel : application au contexte touristique
}

Résumé : Cette recherche questionne l'efficacité des stratégies d'enrichissement des offres d'expérience. Un modèle intégrant différents effets de ces stratégies sur le consommateur : émotions positives élémentaires - enchantement - satisfaction - valeur de consommation intentions comportementales est proposé. Une étude dans le secteur touristique valide empiriquement le modèle mais conduit à rejeter l'hypothèse d'un effet des contextes expérientiels différenciés sur ce modèle.

Mots clés : émotions élémentaires, enchantement, expérience de consommation, marketing expérientiel, satisfaction, valeur

\section{Modeling and testing the impacts of an experiential enrichment strategy: the case of a touristic experience}

\begin{abstract}
This research questions the effectiveness of enrichment strategies on experiential offers. A model accounting for the various effects of these strategies on consumer: primary positive emotions - delight - satisfaction - consumption value - behavioral intentions is suggested. A study in the field of tourism empirically validates the model but does not indicate an impact of the different experiential contexts considered.
\end{abstract}

Keywords: consumption experience, delight, experiential marketing, primary emotions, satisfaction, value 


\section{INTRODUCTION}

Depuis plusieurs années, l'approche expérientielle est considérée par les praticiens comme une source de différenciation majeure dans un contexte de banalisation de l'offre commerciale (Roederer, 2012). Ce positionnement a été adopté dans des secteurs aussi variés que la distribution (L’Occitane), l'exploitation cinématographique (le multiplexe EuropaCorp), le champ muséal (la Cité des Sciences et de l’Industrie) ou encore la gastronomie (le restaurant de G. Blanc à Vonnas). Ces exemples de contextes expérientiels contrôlés par les entreprises sont destinés à proposer aux consommateurs des événements mémorables afin de susciter chez eux des réactions positives et de favoriser à plus long terme leur fidélité (Borghini et al, 2009 ; Caru et Cova, 2006). Cependant, les difficultés, voire les échecs commerciaux, de certaines entreprises proposant à leurs clients des lieux de consommation fortement théâtralisés (Abercrombie \& Fitch, Carrefour Planet, Vulcania, Hard Rock Café), ont conduit les praticiens à s’interroger sur la pertinence de ces stratégies et sur les écarts potentiels entre les intentions liées à ces contextes expérientiels et les réactions effectives des consommateurs (Bäckström et Johansson, 2006).

En conséquence, des travaux scientifiques ont cherché à analyser les effets de ces stratégies expérientielles sur le comportement du consommateur. Dans cette perspective, les émotions élémentaires des individus (Ladhari, 2007) et des concepts comme le ravissement/l'enchantement (Kumar, Olshavsky et King, 2001), la satisfaction (Rust et Oliver, 2000) ou encore la valeur (Holbrook, 1999) ont été mobilisés. La multiplication de ces concepts souligne l'importance accordée par la communauté scientifique à l'analyse de ces effets. Mais une proximité théorique entre les concepts, couplée à des résultats contradictoires, ont entraîné une certaine confusion et font émerger la nécessité de développer un modèle théorique intégrateur des effets de ces stratégies d'enrichissement expérientiel sur le comportement du consommateur.

Cette note de recherche propose donc de répondre à cette double problématique managériale et académique. Tout d'abord, un bilan critique des travaux consacrés aux effets générés par ces stratégies conduit à proposer, au regard du caractère incomplet de chaque concept, un modèle théorique intégrateur. Les concepts retenus ainsi que leurs relations sont justifiés et les effets des contextes expérientiels sur le modèle sont envisagés. Les résultats du modèle testé dans le cadre d'une étude empirique appliquée au secteur du tourisme sont ensuite discutés. Enfin, les apports, limites et voies de recherche sont exposés. 


\section{REVUE DE LA LITTERATURE}

\section{Impact des contextes expérientiels sur le consommateur}

L’impact des contextes expérientiels sur le consommateur a été appréhendé essentiellement au travers des émotions et des évaluations post-consommatoires (Lee et al, 2008). En effet, les émotions positives sont un des effets les plus souvent retenus par les chercheurs pour qui l'expérience de consommation est avant tout comprise comme génératrice de plaisir (Carù et Cova, 2003). Parallèlement, les évaluations développées par le consommateur à la suite de l'expérience vécue ont été étudiées en mobilisant les concepts de satisfaction et de valeur (Anteblian, Filser et Roederer, 2013) afin de mesurer des effets moins transitoires de l’expérience.

\section{Les émotions positives élémentaires et complexes}

Les émotions positives sont souvent considérées comme la première et, pour certains, comme la principale réponse des consommateurs confrontés à des offres d'expériences (Fornerino, Helme-Guizon et Gotteland, 2008 ; Graillot, 1998 ; Mano et Oliver, 1993). Les travaux fondateurs distinguent cependant les émotions élémentaires (primary emotions) des émotions complexes (tertiary emotions) (Izard, 1977 ; Plutchik, 1980).

Concernant les émotions élémentaires, la littérature s’accorde sur la nécessité de disposer d’un large inventaire de réponses afin de capter la richesse des gratifications émotionnelles résultant d'une expérience de consommation (Derbaix et Filser, 2011). Ainsi, soulignant l'absence de véritables instruments permettant de mesurer les émotions ressenties durant les expériences de consommation, Richins (1997) a préconisé l'adoption d'une approche discrète (Consumption Emotion Scale). Plusieurs travaux ont confirmé la nécessité de développer des approches couvrant une variété d'émotions en termes de polarité, d'intensité et de contenu dans le cadre de consommations expérientielles (Faullant, Matzler et Mooradian, 2011 ; Ruth, Brunel et Otnes, 2002). Cependant, si elles permettent de capter des émotions différenciées, leurs mesures posent des difficultés méthodologiques (Laros et Steenkamp, 2005). Au niveau des émotions plus complexes, le concept d'enchantement/ravissement a été proposé (Arnold et al, 2005 ; Finn, 2005 ; Vanhamme, 2008). L'enchantement peut être assimilé à une émotion 
positive extrême ${ }^{1}$, ce concept se situant en aval d'émotions primaires (Kumar, Olshavsky et King, 2001 ; Oliver, Rust et Varki, 1997 ; Rust et Oliver, 2000). Il a été mobilisé à plusieurs reprises afin d'appréhender les émotions ressenties dans le cadre de la consommation de produits avec une forte composante expérientielle comme le tourisme (Magnini, Crotts et Zehrer, 2011 ; St-James et Taylor, 2004) ou la culture (Kumar, Olshavsky et King, 2001 ; Oliver, Rust et Varki, 1997). Mais, si ce concept permet de rendre compte d'émotions intenses et complexes, il reste instable sur le plan conceptuel (St-James et Taylor, 2004).

Parallèlement à ces travaux, d'autres recherches ont tenté de mesurer des effets moins transitoires de l'expérience à travers l'examen des évaluations post-consommatoires.

\section{Evaluation de l'expérience de consommation : satisfaction et valeur}

Partant de l'intuition que le développement de stratégies d'enrichissement expérientiel était susceptible de déboucher sur une plus grande fidélité du consommateur, l'impact des conséquences de l'expérience sur des antécédents de la fidélité - principalement la satisfaction et la valeur (Anteblian, Filser et Roederer, 2013) - a été mesuré.

La satisfaction est «un état psychologique résultant d'une expérience d'achat et/ou de consommation (et donc postérieur à celle-ci) et relatif» (Vanhamme, 2002). La satisfaction a longtemps été associée à des processus cognitifs avant que les modèles explicatifs intègrent les états émotionnels (Mano et Oliver, 1993 ; Wirtz et Bateson, 1999). Ce raffinement conceptuel a permis d'adapter la satisfaction aux expériences vécues dans des contextes théâtralisés (Bigné, Mattila et Andreu, 2008), les outils de mesure conservant - par ailleurs un caractère parcimonieux susceptible de séduire les managers (Vanhamme, 2002). Toutefois, la mesure de la satisfaction ne permet pas de différencier de façon fine les niveaux positifs de satisfaction éprouvés, notamment dans le cas de la consommation de produits expérientiels (Vanhamme, 2008). Par ailleurs, la dimension affective de la satisfaction reste difficile à mesurer (Finn, 2005).

Compte tenu de ces faiblesses, le concept de valeur de consommation a été mobilisé afin d'appréhender l'évaluation de l'expérience par le consommateur. Ce choix se justifie également au regard de l'importance de ce concept, tant sur un plan académique que managérial. Mais, à l’image de la satisfaction, un certain nombre de réaménagements conceptuels a été nécessaire. En effet, la conceptualisation de la valeur s’est longtemps limitée

\footnotetext{
${ }^{1}$ Si la majorité des travaux a assimilé l'enchantement à une émotion extrême (St-James et Taylor, 2004), d'autres auteurs ont considéré ce concept comme une forme de satisfaction extrême (Berman, 2005 ; Torres et Kline, 2006).
} 
à une perspective utilitaire et cognitive comme une valeur d'achat, avant d'être élargie plus récemment à ce qui se passe pendant/après la consommation (Mathwick, Malhotra et Rigdon, 2001). Ce concept permet par combinaison factorielle d'identifier des facettes de la valeur susceptibles d'être perçues à la suite d'une expérience de consommation (Holbrook, 1999). Compte tenu de son caractère multidimensionnel et général, il a été mobilisé pour appréhender des services à fortes composantes expérientielles comme l’hôtellerie, la restauration ou les concerts (Derbaix et Derbaix, 2010 ; De Ruyter et al, 1997 ; SanchezFernandez, Iniesta-Bonillo et Holbrook, 2009). Par comparaison avec la satisfaction, les modèles de la valeur ont l'avantage de proposer des découpages plus fins de l'évaluation de l'expérience par le consommateur (Jones, Reynolds et Arnold, 2006). Ce concept reste néanmoins difficile à opérationnaliser (Sanchez-Fernandez, Iniesta-Bonillo et Holbrook, 2009). Par ailleurs, les mesures de la valeur de consommation varient en fonction du contexte d'étude et débouchent - en conséquence - sur des résultats difficiles à comparer (Walsh, Shiu et Hassan, 2014).

Au final, les concepts analysés conduisent à identifier des effets générés par le déploiement des stratégies expérientielles. Pris un à un, ces concepts présentent de nombreux intérêts mais souffrent de limites théoriques, méthodologiques et/ou managériales et ne peuvent prétendre couvrir l'ensemble des effets produits par l'expérience vécue (tableau 1). En conséquence, il paraît pertinent de proposer un modèle théorique intégrant l’ensemble des effets générés par ces stratégies expérientielles.

[insérer tableau 1]

\section{Proposition d'un modèle intégrateur}

Le rôle des émotions positives élémentaires comme principal antécédent de l'enchantement a été déjà souligné dans différents contextes expérientiels (Loureiro et Kastenholz, 2011 ; Oliver, Rust et Varki, 1997), suggérant ainsi la possibilité d’une hiérarchie entre des émotions élémentaires et complexes (Laros et Steenkamp, 2005 ; Plutchik 1980). Par ailleurs, les aménagements conceptuels autour des modèles d'évaluation de l'expérience ont également conduit à considérer le rôle des émotions positives élémentaires comme source de création de valeur (Babin et Attaway, 2000 ; Duman et Mattila, 2005 ; Hyun, Kim et Lee, 2011) et de satisfaction (Bigné, Andreu et Gnoth, 2005 ; Wirtz, Mattila et Tan, 2000). Enfin, il convient de mentionner que les émotions positives ressenties dans des contextes expérientiels variés (parc à thème, cinéma, spectacle) sont susceptibles d’affecter positivement les intentions de 
retour (Bigné, Andreu et Gnoth, 2005 ; Bigné, Mattila et Andreu, 2008 ; Hume et Mort, 2010). En conséquence, nous postulons que :

$\mathrm{H} 1_{\mathrm{a}, \mathrm{b}, \mathrm{c}, \mathrm{d}}$ : (a) Les émotions positives élémentaires ressenties lors de l'expérience vécue influencent positivement l'enchantement, (b) la valeur de consommation, (c) la satisfaction et (d) les intentions comportementales du consommateur.

Parallèlement, le poids de l'enchantement dans la formation de la valeur et de la satisfaction a été montré (Chen et Chen, 2010 ; Hosany et Gilbert, 2010 ; Yau et Kwong, 2007). En tant qu'émotion positive extrême, l'enchantement, dans le cadre de la consommation touristique, est également susceptible d'expliquer les intentions de retour (Kim, Vogt et Knutson, 2013 ; Loureiro et Kastenholz, 2011). En conséquence, nous proposons que :

$\mathrm{H} 2_{\mathrm{a}, \mathrm{b}, \mathrm{c}}$ : L'enchantement ressenti lors de l'expérience vécue influence positivement (a) la valeur de consommation, (b) la satisfaction et (c) les intentions comportementales du consommateur.

Le rôle de la valeur de consommation comme antécédent de la satisfaction a également été souligné. Si certains auteurs ont validé le lien valeur de consommation - satisfaction (Chen et Chen, 2010 ; Cronin, Brady et Hult, 2000), d'autres ont indiqué l'existence d'effets d'influence partielle de la valeur de consommation compte tenu de son caractère multidimensionnel (Derbaix et Derbaix, 2010). Le fort pouvoir prédictif de la valeur sur les intentions comportementales futures - par contraste avec la satisfaction - a aussi été souligné (Chen et Hu, 2010 ; Cronin, Brady et Hult, 2000 ; Duman et Mattila, 2005 ; Yoon, Lee et Lee, 2010). Ainsi, nous postulons que :

$H 3_{\mathrm{a}, \mathrm{b}}$ : La valeur de consommation retirée de l'expérience vécue influence positivement (a) la satisfaction et (b) les intentions comportementales du consommateur.

Enfin, il a été montré qu'un score de satisfaction élevé conduit à des intentions de retour plus fortes dans une variété de contextes expérientiels tels que les parcs à thème (Bigné, Mattila et Andreu, 2008), les croisières (Duman et Mattila, 2005), les sites patrimoniaux (Chen et Chen, 2010) ou les concerts de variété (Derbaix et Derbaix, 2010). Dès lors, nous proposons que :

H4 : La satisfaction retirée de l'expérience vécue influence positivement les intentions comportementales.

A l'image des modèles intégrateurs de prise de décision appliqués aux produits expérientiels (Dynamic Model of Affective Response de Cohen et Areni, 1991 ; Consciousness Emotion Value Model d'Holbrook, 1986 ; Experiential Decision Model de Kwortnik et Ross, 2007), ces hypothèses permettent de proposer un modèle articulant l'ensemble des effets générés par 
un contexte expérientiel sur le consommateur (figure 1). Plus particulièrement un modèle intégrateur offre les avantages de pouvoir clarifier :

- l'existence de différents concepts, certains étant proches théoriquement ;

- la place de chacune de ces variables au sein d'un processus séquentiel d’influence de l’expérience sur le consommateur ;

- les relations (polarité et intensité) entre ces différentes variables.

[insérer figure 1]

Impact des contextes expérientiels sur le consommateur: l'effet différencié de la théâtralisation

Les tentatives de réenchantement du système marchand, à travers la proposition d'offres immersives, souvent hyperréelles et spectaculaires, se sont multipliées sur le postulat que l'enrichissement des offres d'expérience permettrait de générer chez le consommateur des effets positifs (Pine et Gilmore, 1998 ; Ritzer, 2005 ; Schmitt, 1999). Managers et chercheurs se sont accordés sur la proposition d'expériences extraordinaires en les considérant, par contraste avec des expériences ordinaires, comme génératrices d’émotions additionnelles et susceptibles d'accroître significativement la valeur, la satisfaction et la fidélité (Badot, 2005 ; Remy, 2007). Dans cette perspective, la théâtralisation - ou la mise en scène du lieu par un travail important sur le décor - est le levier le plus efficace afin de matérialiser le positionnement des contextes expérientiels contrôlés et d'enrichir l'expérience vécue par le consommateur (Filser, 2002 ; Kreziak et Frochot, 2011). Cependant, à l'instar de Sands, Oppewal et Beverland (2009), force est de constater le faible nombre d'études empiriques permettant de valider cette affirmation. Il convient cependant de noter qu'aucune recherche n’a, par approche comparée, montré un effet différencié d’un contexte expérientiel théâtralisé. Cet effet peut cependant être supposé et conduit à proposer l’hypothèse suivante :

H5 : Une offre d’expérience théâtralisée aura un impact significativement plus élevé qu'une expérience non théâtralisée sur les relations existant entre les variables du modèle intégrateur.

\section{METHODOLOGIE}

\section{Contextes expérientiels sélectionnés}

Le modèle et les hypothèses associées ont fait l'objet d'une validation empirique dans le domaine du tourisme et plus particulièrement dans le cadre de séjours en station de ski. Ce 
champ d'investigation a été retenu en raison des nombreux enjeux liés au management de l'expérience client pour les professionnels du tourisme (Chen et Chen, 2010). Il s'agit par ailleurs d’un des domaines initialement privilégiés par l'approche expérientielle (Giannelloni, 2011 ; Shaw, Bailey et Williams, 2011). Cette étude porte ainsi sur la station des Arcs et plus particulièrement sur Arc 1800 et Arc 1950 (encadré 1). La collecte sur deux sites du même domaine skiable permet de comparer deux offres ayant des stratégies expérientielles différenciées (théâtralisée versus non théâtralisée) et d’atténuer les effets du domaine (pistes de ski, espace naturel) sur les réponses des enquêtés. A l'instar de Dolbec et Chebat (2013), cette démarche peut être qualifiée de quasi-expérimentale.

\section{[insérer encadré 1]}

Les consommateurs ont été interrogés sur une période de deux semaines, au cours du mois d'avril 2009, à l'exclusion des samedi et dimanche (jours d'arrivée et de départ des clients). Les conditions climatiques (temps ensoleillé et enneigement satisfaisant) sont restées stables lors de la période de collecte. L’échantillon final est composé de 297 touristes résidant à Arc 1800 et de 244 résidant à Arc 1950. Les consommateurs ont été exclusivement interrogés à propos de leur expérience dans leur station de résidence. Aucune différence significative n’apparaît entre les structures des deux échantillons à l'exception du niveau de rémunération qui peut s’expliquer par le positionnement haut de gamme d'Arc 1950 (A1).

\section{Echelles de mesure mobilisées}

Les échelles de mesure utilisées se réfèrent à des instruments développés antérieurement. Les construits multidimensionnels complexes (émotions positives élémentaires et valeur de consommation) ont toutefois été adaptés au contexte de la consommation touristique au moyen d'études pilotes (A2).

Ainsi, les émotions ressenties ont été mesurées au moyen du Consumption Emotions Set de Richins (1997), décliné en France par Ferrandi, De Barnier et Valette-Florence (2002). Cet instrument de mesure a été choisi pour ses qualités sur les plans théorique et psychométrique. Il permet en effet de couvrir la plupart des réactions affectives rencontrées dans le champ de la consommation (Derbaix et Filser, 2011) et a montré sa supériorité (Richins, 1997) sur d'autres outils tels que le DES (Izard, 1977), l'EPI (Plutchik et Kellerman, 1974) ou le PAD (Mehrabian et Russell, 1974). La mesure a été adaptée à la situation de consommation étudiée, comme suggéré par Laros et Steenkamp (2005). Les études réalisées ont conduit à retenir 3 émotions mesurées par 7 items (A2). 
De même, en s'appuyant sur les travaux ayant développé des mesures de la valeur de consommation dans des contextes expérientiels variés (Aurier, Evrard et N’Goala, 2004 ; Derbaix et Derbaix, 2010 ; Mathwick, Malhotra et Rigdon, 2001) et suite à deux études pilotes (A2), 10 dimensions de la valeur ont été retenues afin de mesurer la valorisation par les consommateurs du séjour vécu dans une station de ski.

Par ailleurs, à l'instar de Finn (2005) et Loureiro (2010), l'enchantement a été mesuré par trois items. La satisfaction a été appréhendée au moyen de deux items (Kim, Vogt et Kutson, 2013). Enfin, les intentions futures ont été appréciées par un item mesurant l'intention de revenir dans la station de ski.

Pour chaque item proposé, les consommateurs devaient indiquer un degré d’intensité (émotions), d'accord (enchantement, satisfaction et valeur) ou de probabilité (intentions) en utilisant une échelle de type Likert en six échelons.

\section{RESULTATS}

\section{Qualités psychométriques des construits}

Des analyses factorielles confirmatoires ont été réalisées sur les données collectées au moyen des équations structurelles et plus spécifiquement de la méthode des moindres carrés partiels $\left(\mathrm{PLS}^{2}\right.$ ) avec une procédure de bootstrap de 200 itérations (Tenenhaus et al, 2005). Elles ont permis de valider les structures factorielles supposées des construits, en cohérence avec les travaux antérieurs (enchantement et satisfaction) ou avec les analyses factorielles exploratoires précédemment réalisées (émotions positives élémentaires et valeur de consommation). Par ailleurs, les poids factoriels (loadings), supérieurs à 0,50 et statistiquement significatifs au seuil de 1\%, sont satisfaisants (A3).

Des facteurs de second ordre ont été demandés pour quatre construits : les émotions positives élémentaires (Richins, 1997), la valeur plaisir (Malhotra, Matwick et Rigdon, 2001), la valeur sociale (Sweeney et Soutar, 2001) et la valeur de distinction (Walsh, Shiu et Hassan, 2014) (A4). Les niveaux des corrélations obtenues entre les facteurs de premier ordre lors des analyses factorielles confirmatoires valident cette démarche. Celle-ci permet d’obtenir les

\footnotetext{
${ }^{2}$ La méthode PLS a été privilégiée dans cette recherche car : $1^{\circ}$ elle permet de traiter des modèles relationnels comportant un grand nombre de variables; $2^{\circ}$ elle ne nécessite pas la multinormalité des variables ; $3^{\circ}$ elle est particulièrement adaptée au test de modèles externes - par opposition à la modélisation interne (Vilares, Almeida et Coelho, 2010).
} 
structures factorielles à privilégier, comme l'ont initialement préconisé les auteurs des construits mobilisés (A6).

Des analyses multi-groupes et des tests de permutation ont été réalisés. Ils fournissent, pour chaque poids factoriels (loadings) et chaque lien postulé (path coefficient), un test de significativité sur la différence entre les valeurs obtenues pour chaque sous-groupe (Chin et Dibbern, 2010). Ces analyses permettent de conclure à l'invariance totale des mesures utilisées pour les deux échantillons considérés (Arc 1800 et Arc 1950).

Les coefficients Alpha de Cronbach et Rhô de Jöreskog ont ensuite permis d'évaluer la fiabilité des outils de mesure. Les coefficients calculés sont satisfaisants $(>0,7)$ tant pour les facteurs de premier que de second ordre ${ }^{3}$ (A5).

Enfin, l'approche proposée par Fornell et Larcker (1981) a permis d'établir les validités convergentes et discriminantes (A6).

Les analyses menées permettent de conclure à la fiabilité et à la validité des différents construits pour les deux échantillons considérés. Ce premier résultat apparaît comme significatif au regard de la proximité théorique de certains concepts (enchantement, satisfaction) et de la difficile opérationnalisation d'autres variables (émotions positives élémentaires, valeur de consommation). Il est également satisfaisant compte tenu des problèmes de validité discriminante précédemment soulignés pour ces construits (Vanhamme, 2008).

\section{Tests du modèle de recherche}

Suite aux tests des modèles de mesure, l'ensemble des liens du modèle de recherche proposé a été testé avec PLS et une procédure de bootstrap de 200 réplications. Les indices d'ajustement fournis par l'analyse multi-groupes réalisée pour le GoF externe (performance des modèles de mesure) et le GoF interne (performance des modèles structurels) sont respectivement de 0,994 et 0,826 (Arc 1800) et de 0,997 et 0,841 (Arc 1950). L'ajustement des modèles de mesure et structurels proposés est donc satisfaisant. L'examen des valeurs des paramètres et de leur significativité met en évidence des relations causales entre les construits mesurés (tableau 2).

[insérer tableau 2]

Dans un premier temps, le rôle des émotions positives élémentaires est examiné (H1). Les résultats indiquent qu'elles expliquent près de $50 \%$ de l'enchantement des touristes lors de

\footnotetext{
${ }^{3}$ Excepté pour la valeur de pratique sociale pour Arc 1800.
} 
leur séjour et conduisent à accepter l'hypothèse H1a. La pertinence de l'articulation entre des émotions élémentaires (la stimulation, la joie et la tranquillité) et une émotion subséquente positive et extrême (l'enchantement) est ainsi confirmée, au contraire des travaux de Barnes, Beauchamp et Webster (2010) où la joie pouvait suffire à générer de l'enchantement. L'examen des liens structurels montre également une influence positive et significative des émotions positives élémentaires sur les valeurs fonctionnelle (Arc 1800 et Arc 1950), de plaisir (Arc 1800 et Arc 1950) et de distinction (Arc 1950) ainsi qu'une absence d'influence sur la valeur sociale. Si ces résultats amènent à valider partiellement $\mathrm{H} 1 \mathrm{~b}$, ils confirment cependant que ces émotions élémentaires jouent un rôle dans la formation de la valeur de consommation (Babin et Attaway, 2000). Les émotions positives élémentaires expliquent aussi la satisfaction éprouvée par les touristes lors de leur séjour (Arc 1800 et Arc 1950), validant ainsi H1c. Les émotions positives élémentaires sont donc une variable explicative du niveau de satisfaction des consommateurs (Fornerino, Helme-Guizon et Gotteland, 2008). Enfin, l’influence des émotions positives élémentaires sur les intentions comportementales des consommateurs à l'égard de la station de ski (Arc 1800 et Arc 1950) est non significative (H1d). Si ce résultat est contre intuitif au regard du modèle approche / évitement (Mehrabian et Russell, 1974), il vient cependant alimenter la difficulté d'expliquer les intentions futures du consommateur à l'égard d'une destination touristique, par contraste avec des offres d'expérience telles que les parcs d'attraction (Chi et Qu, 2008).

Dans un second temps, les effets de l'enchantement sur la valeur, la satisfaction et les intentions comportementales sont envisagés (H2). Ainsi, l'enchantement influence positivement et significativement trois valeurs de consommation (valeur plaisir, de distinction et sociale), la satisfaction ainsi que les intentions comportementales. Son impact est en revanche non significatif sur la valeur fonctionnelle. Les résultats étant homogènes pour les deux contextes, H2a est partiellement acceptée tandis que H2b et H2c sont validées. Si l'enchantement a déjà fait l'objet de développements dans la littérature, le faible nombre d'études empiriques invite à interpréter les résultats obtenus avec prudence (Vanhamme, 2008). Cependant, ces résultats sont intéressants car ils confirment que l'enchantement, comme émotion positive extrême, a un effet causal post-consommatoire dans le cadre d'une consommation à dominante expérientielle (Vanhamme, 2008). Ils montrent également que cet effet s'exerce tout au long de la chaîne de causalité - aucun des travaux antérieurs ne montrant l'existence d'une telle articulation.

En remontant la chaîne de causalité du modèle intégrateur, l’hypothèse H3 permet d'étudier l'influence de la valeur de consommation sur la satisfaction et les intentions 
comportementales. Concernant le lien valeur - satisfaction, le type de valeur et leur influence dépend de la station étudiée. Ainsi, seule la valeur de distinction a un impact positif et significatif sur la satisfaction pour Arc 1800 tandis que les valeurs sociale et fonctionnelle ont un impact positif et significatif sur la satisfaction des touristes de la station Arc 1950. Les bénéfices associés à chaque offre et perçus par les touristes au cours de l'expérience sont susceptibles d'expliquer ces résultats contrastés. H3a n’est donc que partiellement validée. Outre cette influence partielle, les résultats montrent une absence d'impact des dimensions de la valeur de consommation (valeur de plaisir, sociale, de distinction et fonctionnelle) sur les intentions futures des touristes (Arc 1800 ou Arc 1950). H3b doit donc être rejetée, allant ainsi à l'encontre des travaux précédents (Chen et $\mathrm{Hu}$, 2010). Si la complexité des relations entre ces concepts et le caractère fortement contextuel du concept de valeur amènent à relativiser ces conclusions, elles paraissent intéressantes à deux titres. Elles remettent en cause l’idée que la valeur de consommation est une variable alternative plus séduisante que la satisfaction afin de prédire les intentions futures (Yi et Hoseong, 2003). Par ailleurs, à l'instar d'Aurier, Benavent et N’Goala (2001), il paraît difficile de considérer la valeur hors de tout enchâssement relationnel plus global avec comme seul objectif d’appréhender une expérience isolée.

Enfin, cette recherche permet d'examiner le lien satisfaction-intentions comportementales (H4) qui est positif et significatif pour les 2 échantillons et valide ainsi H4. Il convient de souligner que la satisfaction contribue le plus fortement aux intentions comportementales conjointement avec l'enchantement, ces deux variables expliquant près de $40 \%$ des intentions de retour des consommateurs. Ce résultat confirme l'intérêt de continuer à utiliser la satisfaction dans des modèles de comportement déclinés dans des contextes expérientiels (Kim, Vogt et Knutson, 2013).

En complément du test de ces hypothèses, des tests de médiation ont été menés pour chaque échantillon (Arc 1800 et Arc 1950) afin d'affiner le rôle joué par l'enchantement, la valeur de consommation et la satisfaction au sein de cette séquence de causalité. Ils ont été réalisés en spécifiant simultanément les effets directs et indirects (introduction des différents médiateurs) (A7). Si les effets directs deviennent non significatifs lorsque la variable médiatrice est intégrée, cela indique un effet de médiation totale selon Baron et Kenny (1986) ou indirecte selon Zhao, Lynch et Chen (2011). Si les effets directs et indirects coexistent, la médiation est partielle selon Baron et Kenny (1986) ou complémentaire selon Zhao, Lynch et Chen (2011). Les analyses réalisées confirment le rôle pivot de l'enchantement qui exerce un effet de médiation totale (relation émotions élémentaires - valeur de consommation et relation 
émotions élémentaires - intentions futures) ou partielle (relation émotions élémentaires valeur de consommation et relation émotions élémentaires - satisfaction) tout au long de la chaîne de causalité 4 . Elles soulignent également le rôle de médiateur total (relation émotions élémentaires - intentions futures et relations valeur de consommation - intentions futures) ou partiel (relation enchantement - intentions futures) de la satisfaction. Inversement, la valeur de consommation n'exerce pas d'effet de médiation au sein de la chaîne de causalité ${ }^{5}$.

Cette recherche confirme ainsi l'existence d'un processus séquentiel d'effets de l'expérience sur le consommateur. Elle souligne le rôle déterminant des émotions positives élémentaires en raison de leur influence sur la valeur de consommation et sur la satisfaction. Cependant, cette influence est en partie médiatisée par l'enchantement qui joue un rôle clef dans cette chaîne de causalité. Ce concept exerce par ailleurs une influence directe sur la satisfaction et les intentions futures. Par contraste, la valeur de consommation n'exerce que des effets d'influence partielle et ne joue pas de rôle médiateur dans la chaîne de causalité. Enfin, la satisfaction reste, dans le cadre de la consommation d'offres d'expérience, le meilleur prédicteur des intentions futures.

Le modèle proposé ainsi validé, il reste à apprécier les effets différenciés de la stratégie d’enrichissement expérientiel déployée par Arc 1800 (non théâtralisée) par contraste avec Arc 1950 (théâtralisation). Compte tenu du positionnement voulu des deux sites touristiques, il est postulé des offres d'expérience différentes et donc des effets différenciés sur le consommateur (Dolbec et Chebat, 2013).

Des comparaisons de moyennes pour échantillons indépendants indiquent préalablement des différences significatives pour les niveaux d'émotions élémentaires, d’enchantement et de satisfaction (supérieurs pour Arc 1950 ; p < 0,01). Cependant, l'analyse multi-groupes réalisée ne montre pas, hormis pour le lien valeur fonctionnelle-satisfaction (non significatif pour Arc 1800 et significatif au seuil de 1\% pour Arc 1950), de différences significatives entre les deux contextes expérientiels étudiés. Bien que le modèle épuré ${ }^{6}$ proposé dans cette recherche soit stable pour les deux stations (figures 2 et 3), les analyses réalisées conduisent à

\footnotetext{
${ }^{4}$ L'enchantement est un médiateur total de la relation émotions élémentaires - valeur sociale (Arc 1800 et Arc 1950) et de la relation émotions élémentaires - valeur de distinction (Arc 1800). L'enchantement est un médiateur partiel de la relation émotions élémentaires - valeur plaisir (Arc 1800 et Arc 1950) et de la relation émotions élémentaires - valeur de distinction (Arc 1950).

${ }^{5}$ Excepté pour la valeur fonctionnelle qui est un médiateur partiel de la relation émotions élémentaires satisfaction (Arc 1950).

${ }^{6}$ Pour chaque échantillon, tous les liens non-significatifs ont été supprimés.
} 
rejeter H5. Par comparaison avec Arc 1800, si l'expérience proposée à Arc 1950 génère notamment des émotions plus fortes, ces dernières n’impactent pas de façon plus significative la valeur, la satisfaction et la fidélité. Ainsi, contrairement au postulat défendu par Pine et Gilmore (1998) et Schmitt (1999), les données recueillies ne permettent pas d'affirmer un effet différencié de la théâtralisation mise en œuvre par Arc 1950 - par contraste avec Arc 1800.

[insérer figures 2 et 3]

\section{APPORTS, LIMITES ET VOIES DE RECHERCHE}

\section{Apports de la recherche}

La proposition, le test et la validation d'un modèle intégrant les différents effets de l'expérience sur les consommateurs constituent la première contribution théorique de ce travail. Il offre la possibilité d’appréhender la polarité, l'intensité et la nature extrêmement variée de ces effets. Les émotions élémentaires positives, l'enchantement, la valeur de consommation et la satisfaction du consommateur sont autant de réponses individuelles mobilisées par les chercheurs pour appréhender l'expérience vécue. Toutefois, ces concepts pris séparément - ne restituent qu’imparfaitement la richesse de l'expérience vécue. Parallèlement, la littérature plaide en faveur du développement de modèles intégrateurs (Gallarza, Gil-Saura et Holbrook, 2011 ; Kwortnik et Ross, 2007). La proposition d'un tel modèle théorique clarifie la place de chaque concept au sein d'une chaîne de causalité articulant différentes réactions émotionnelles, des évaluations post-consommatoires et des intentions futures.

Parallèlement à cette investigation théorique, la phase de validation empirique permet de distinguer ces concepts en vérifiant leur validité discriminante et de comprendre leur rôle au cours de l'expérience vécue. Dans un premier temps, le processus séquentiel conduit à souligner l'influence centrale d'états émotionnels primaires ou complexes (enchantement) sur les évaluations post-consommatoires (satisfaction et fidélité). Par la suite, à l'exception de l'enchantement, ces états émotionnels ne semblent pas déterminer les intentions futures. Ce résultat atteste à la fois de l’importance et de la complexité des effets générés par les émotions ressenties tout au long de la chaîne de causalité. Afin d’appréhender ces effets, il paraît nécessaire d'articuler différentes conceptualisations de réactions émotionnelles, à l'instar du lien proposé ici entre émotions primaires et émotions subséquentes (Laros et Steenkamp, 2005). Dans un second temps, le test du modèle conduit à souligner concomitamment le rôle 
limité de la valeur de consommation et celui central de la satisfaction comme déterminant des intentions futures. La très bonne capacité prédictive de la satisfaction peut s’expliquer par les aménagements conceptuels dont elle a fait l'objet (Vanhamme, 2008). Parallèlement, la faible influence de la valeur sur la satisfaction et les intentions comportementales peut trouver sa justification dans les difficultés associées à son opérationnalisation. Son utilisation pour appréhender les effets d'une expérience soulève également des interrogations au regard de son caractère intrinsèquement dynamique (Holbrook, 1999). Il s'agit cependant d'un concept qui par contraste avec celui de satisfaction - permet de proposer une description plus fine des effets de l'expérience sur les évaluations post-consommatoires (Chen et Chen, 2010). Ainsi, une position conciliatrice mobilisant conjointement valeur de consommation et satisfaction pour appréhender les évaluations réalisées par le consommateur paraît pertinente (Oliver, 1999).

Un second apport théorique réside dans l'approche comparative mise en œuvre qui permet de mieux cerner les effets d'un contexte expérientiel contrôlé par l'entreprise sur le consommateur. Sur ce point, les résultats conduisent à rejeter l'idée d'un effet positif de la théâtralisation. Cette dernière ne génère pas d'effets additionnels à court terme sur les réactions émotionnelles primaires ou l'enchantement et ultérieurement sur les jugements postconsommatoires et les intentions de fidélité. Contrairement à ce que suggère la littérature, l'offre proposant un environnement théâtralisé (Arc 1950) n’influence pas significativement les schémas de réaction, d'évaluation et d'intention. Ces résultats remettent en question la pertinence des stratégies d'enrichissement expérientiel du point de vue de leur réception par le consommateur (Bäckström et Johansson, 2006). Si l'enthousiasme des praticiens pour cette orientation stratégique reste important malgré les échecs commerciaux, cette note de recherche montre que l'enrichissement expérientiel, matérialisé ici par la théâtralisation, n’engendre pas de réponses positives supplémentaires de la part des consommateurs.

Sur un plan managérial, ces résultats interrogent l'intérêt d'investir massivement dans des dispositifs destinés à enchanter l'offre d'expérience touristique. Compte tenu des enjeux concurrentiels au sein de l'industrie touristique et des difficultés rencontrées actuellement par les stations de sports d'hiver françaises pour fidéliser leur clientèle (Goncalvès et al, 2011), l’orientation stratégique explorée par Arc 1950 a pu apparaître comme une réponse pertinente. Cependant, si ces formes d'enrichissement expérientiel ont des potentiels d'attraction particulièrement élevés, notamment auprès des primo visiteurs (Roederer, 2012), elles ne semblent pas en capacité de générer des expériences plus riches sur le plan émotionnel, plus valorisées et plus satisfaisantes. Ce résultat mérite d'être souligné au regard notamment des 
investissements massifs que ces stratégies engendrent. A titre d'illustration, un investissement de 250 millions d’euros a été nécessaire afin de développer Arc 1950 avant son ouverture en 2008. Les stations françaises pourraient envisager d'autres stratégies - peut-être moins coûteuses - à l'instar de la station de Val Thorens (Savoie) qui a opté pour un positionnement orienté vers le partage et l'esprit communautaire. La station s'appuie notamment sur la proposition d'évènements attractifs et sur un véritable marketing de destination. Par ailleurs, au-delà des effets d'attraction, les praticiens doivent s'assurer a minima que l'enrichissement de l'offre génère des effets additionnels non négligeables auprès du consommateur. Ce défi managérial requiert une connaissance relativement fine des caractéristiques des clients et de leurs schémas comportementaux. Dans cette perspective, le modèle intégrateur proposé constitue un outil apte à rendre compte de ces effets.

\section{Limites et perspectives}

Une première limite touche à la validité externe de la recherche. Les résultats sont contingents au contexte de la recherche (deux stations de ski appartenant au même domaine skiable). Dans cette perspective, il paraît nécessaire d'envisager le test de ce modèle intégrateur dans des contextes touristiques proches (stations de ski ayant adopté des stratégies similaires à l’image de Whistler au Canada ou de Vail aux Etats-Unis), dans d'autres contextes touristiques qui proposent des offres d'expériences enrichies (parcs ludo-éducatifs comme le Paléosite, parcs animaliers comme Micropolis ou parcs aquatiques comme Aqualand) voire dans des contextes plus éloignés (à l’image de la distribution).

Il faut également remettre en perspective l'absence d'effet différencié constaté entre les deux stations. La stratégie d'enrichissement expérientiel adoptée par Arc 1950 concerne essentiellement le village et son caractère fortement théâtralisé. Or, une station de ski est un système touristique complexe qui regroupe sur un même site plusieurs offres (pistes de ski, offres d'hébergement et de restauration, autres activités de loisirs, etc.) (Kreziak et Frochot, 2011). Dans cette perspective, sans être un élément marginal, la théâtralisation du village apparaît comme un élément périphérique de l’offre globale de séjour en station de ski. Par ailleurs, le produit touristique est un produit intrinsèquement expérientiel (Giannelloni, 2011). Même si les possibilités d'accentuer cette particularité sont nombreuses, la stratégie d'enrichissement expérientiel doit être relativisée. Ces deux facteurs peuvent expliquer l'absence d'écart constaté entre les deux stations sur le modèle intégrateur. Dans cette perspective, trois voies de recherche peuvent être envisagées. Il serait intéressant d'examiner dans des logiques comparatives des systèmes d'offre où la stratégie d'enrichissement 
expérientiel touche plus directement le cœur de l’offre. Ce degré de centralité est susceptible d'affecter plus fortement l'ensemble des schémas du consommateur. Dans le prolongement de cette première proposition, il serait également pertinent d'apprécier l'impact de différents éléments de l'offre enrichie sur l'ensemble du modèle intégrateur. Par contraste avec l’approche globale développée ici (le séjour en station de ski), le découpage analytique d'un système d'offre (par exemple offre centrale/offre périphérique) pourrait conduire plus facilement à la mise au jour d'effets différenciés de ces offres d'expérience. Enfin, mener une étude longitudinale constituerait une voie de recherche intéressante afin d'apprécier, dans le temps, les effets de stratégies d'enrichissement expérientiels contrastées sur l'ensemble des variables considérées.

Il convient également de remettre en perspective le modèle intégrateur proposé. Tout d'abord, le statut de certaines variables et de certains liens mérite d'être discuté. Ainsi, l'enchantement a été appréhendé comme une émotion alors qu'il est assimilé par d'autres chercheurs à une forme de satisfaction extrême (Berman, 2005 ; Torres et Kline, 2006). De même, l'articulation entre valeur de consommation et satisfaction pourrait être réexaminée au regard du caractère non récursif de la relation (Oliver, 1999). Ces interrogations théoriques laissent entrevoir la possibilité de modifier la position de certaines variables dans la chaîne de causalité. Par ailleurs, la validité prédictive du modèle a été appréciée en mobilisant les intentions comportementales. Il ne s'agit cependant que d'une approximation éloignée du comportement de fidélité (Frisou, 2005). L’introduction de concepts tels que la confiance, l'attachement et le comportement réel de fidélité devrait permettre d'appréhender de manière plus exhaustive l’impact de l'expérience sur la fidélité du consommateur. Enfin, certaines variables ont été ignorées à l'image de l'immersion (Carù et Cova, 2003 ; Fornerino, Helme-Guizon et Gotteland, 2008 ; Maubisson, 2012) ou du flow (Csikszentmihaly, 1997 ; McGinnis, Gentry et Gao, 2008 ; Tsaur, Yen et Hsiao, 2013). Ce choix s’explique par les ambiguïtés théoriques et méthodologiques conséquentes associées à leurs statuts (état et/ou processus), leurs mesures et leurs relations avec d'autres variables. De même, le modèle ignore la prise en compte de potentiels modérateurs individuels (motivation intrinsèque ou extrinsèque, expérience historique personnelle vis-à-vis de la station, implication à l'égard de l'expérience touristique, sports et loisirs pratiqués, etc.). Ces différentes variables peuvent pourtant s’avérer utiles afin d'enrichir la compréhension des effets de l'expérience sur le consommateur. Ces différentes limites invitent donc les chercheurs à proposer, tester et mettre en concurrence des modèles intégrateurs alternatifs. 


\section{Références bibliographiques}

Anteblian B, Filser M et Roederer C (2013) L'expérience du consommateur dans le commerce de détail. Une revue de littérature. Recherche et Applications en Marketing 28 (3): 84-113.

Arnold MJ, Reynolds KE, Ponder N et Lueg JE (2005) Customer delight in a retail context, investigating delightful and terrible shopping experience. Journal of Business Research 58 (8): 1132-1145.

Aurier P, Evrard Y et N'Goala G (2004) Comprendre et mesurer la valeur du point de vue du consommateur. Recherche et Applications en Marketing 19 (3): 1-20.

Aurier P, Benavent C et N'Goala G (2001) Validité discriminante et prédictive des composantes de la relation à la marque. In: Actes $d u 17^{\text {ème }}$ congrès international de l'Association Française du Marketing, Deauville, France, CD-Rom.

Babin BJ et Attaway JS (2000) Atmospheric affect as a tool for creating value and gaining share of customer. Journal of Business Research 49 (2): 91-99.

Bäckström K et Johansson U (2006) Creating and consuming experiences in retail store environments: comparing retailer and consumer perspectives. Journal of Retailing and Consumer Services 13 (6): 417-430.

Badot O (2005) L’autre raison du succès de Wal-Mart: une rhétorique de l'infra-ordinaire. Revue Française du Marketing 203 (3/5): 97-117.

Barnes DC, Beauchamp MB et Webster C (2010) To delight, or not to delight ? This is the question service firms must address. The Journal of Marketing Theory and Practice 18 (3): 275-284.

Baron RM et Kenny DA (1986) Moderator-mediator variables distinction in social psychological research: conceptual, strategic, and statistical considerations. Journal of Personality and Social Psychology 51 (6): 1173-1182.

Berman B (2005) How to delight your customers. California Management Review 48 (1): 129-151. 
Bigné JE, Andreu L et Gnoth J (2005) The theme park experience: an analysis of pleasure, arousal and satisfaction. Tourism Management 26 (6): 833-844.

Bigné JE, Mattila AS et Andreu L (2008) The impact of experiential consumption cognitions and emotions on behavioral intentions. Journal of Services Marketing 22 (4): 303-315.

Borghini S, Diamond N, Kozinets RV, McGrath MA, Muñiz AM Jr et Sherry JF Jr (2009) Why are themed brand stores so powerful ? Retail brand ideology at American Girl Place. Journal of Retailing 85 (3): 363-375.

Carù A et Cova B (2003) Approche empirique de l'immersion dans l'expérience de consommation : les opérations d'appropriation. Recherche et Applications en Marketing 18 (2): 47-65.

Carù $\mathrm{A}$ et Cova B (2006) Expériences de marque : comment favoriser l'immersion du consommateur. Décisions Marketing 41 (janvier-mars): 43-52.

Chen CF et Chen FS (2010) Experience quality, perceived value, satisfaction and behavioral intentions for heritage tourists. Tourism Management 31 (1): 29-35.

Chen PT et Hu HH (2010) How determinant attributes of service quality influence customerperceived value: an empirical investigation of the Australian coffee outlet industry. International Journal of Contemporary Hospitality Management. 22 (4): 535-551.

Chi CGQ et Qu H (2008) Examining the structural relationships of destination image, tourist satisfaction and destination loyalty: an integrated approach. Tourism management 29 (4): 624636.

Chin WW et Dibbern J (2010) An introduction to a permutation based procedure for multigroup PLS analysis: results of tests of differences on simulated data and a cross cultural analysis of the sourcing of information system services between Germany and the USA. In: Vinzi VE, Chin WW, Henseler J et Wang H (coord.) Handbook of partial least squares concepts: concepts, methods and applications. New York: Springer Handbooks of Computational Statistics, pp.171-193.

Cohen JB et Areni CS (1991) Affect and consumer behavior. Handbook of Consumer Behavior 4 (7): 188-240. 
Cronbach LJ (1951) Coefficient alpha and the internal structure of tests. Psychometrica 16 (3): 297-334.

Cronin JJ Jr, Brady MK et Hult GTM (2000) Assessing the effects of quality, value, and customer satisfaction on consumer behavioral intentions in service environments. Journal of Retailing 76 (2): 193-218.

Csikszentmihalyi M (1997) Finding flow: The psychology of engagement with everyday life. New York: Basic Books.

Derbaix M et Derbaix C (2010) Les tournées du souvenir: des générations en quête d'authenticité ? Recherche et Applications en Marketing 25 (3): 57-84.

Derbaix C et Filser M (2011) L'affectif dans les comportements d'achat et de consommation. Paris: Economica, Gestion.

de Ruyter K, Wetzels M, Lemmink J et Mattson J (1997) The dynamics of the service delivery process: a value-based approach. International Journal of Research in Marketing 14 (3): 231-243.

Dolbec PY et Chebat JC (2013) The Impact of a flagship vs a brand store on brand attitude, brand attachment and brand equity. Journal of Retailing 89 (4): 460-466.

Duman T et Mattila AS (2005) The role of affective factors on perceived cruise vacation value. Tourism Management 26 (3): 311-323.

Faullant R, Matzler K et Mooradian TA (2011) Personality, basic emotions, and satisfaction: primary emotions in the mountaineering experience. Tourism Management 32 (6): 1423-1430.

Ferrandi JM, De Barnier V et Valette-Florence P (2002) Une première application de l'échelle de Richins pour mesurer les réactions émotionnelles à la publicité. In: Actes du $18^{\text {ème }}$ congrès international de l'Association Française du Marketing, Lille, France, 1-29.

Filser M (2002) Le marketing de la production d'expérience. Statut théorique et implications managériales. Décisions Marketing 28 (Octobre): 13-22.

Finn A (2005) Reassessing the foundations of customer delight. Journal of Service Research 8 (2): 103-116. 
Firat AF et Ulusoy E (2011) Living a theme. Consumption Markets \& Culture 14 (2): 193202.

Fornell C et Larcker DF (1981) Evaluating structural equation models with unobservable variables and measurement error. Journal of Marketing Research 18 (1): 39-50.

Fornerino M, Helme-Guizon A et Gotteland D (2008) Expériences cinématographiques en état d'immersion: effets sur la satisfaction. Recherche et Applications en Marketing 23 (3): 95113.

Frisou J (2005) Une approche tendancielle du comportement de fidélité: du concept à sa mesure. Recherche et Applications en Marketing 20 (2): 105-125.

Gallarza MG, Gil-Saura I et Holbrook MB (2011) The value of value: further excursions on the meaning and role of customer value. Journal of Consumer Behaviour 10 (4): 179-191.

Giannelloni JL (2011) La recherche marketing au service des pratiques touristiques : mythe ou réalité. Décisions Marketing 64 (octobre-décembre): 1-4.

Goncalves O, Guallino G, Michel H et Robinot E (2011) Flocon ou chamois d'or ? Mesurer la performance marketing d'un service touristique. Le cas des stations de ski françaises. Décisions Marketing 64 (octobre-décembre): 59-68.

Graillot L (1998) Emotions et comportement du consommateur. Recherche et Applications en Marketing 13 (1): 5-23.

Hair JF Jr, Anderson RE, Tatham RL et Black WC (1998), Multivariate Data Analysis. $5^{\text {th }}$ edition. Upper saddle River: Prentice Hall.

Holbrook MB (1986) Aims, concepts, and methods for the representation of individual differences in esthetic responses to design features. Journal of Consumer Research 13 (3): 337-347.

Holbrook MB (1999) Consumer Value: a framework for analysis and research. London and New York: Routledge Interpretive Market Research Series.

Hosany S et Gilbert D (2010) Measuring tourists' emotional experiences toward hedonic holiday destinations. Journal of Travel Research 49 (4): 513-526. 
Hume M et Mort GS (2010) The consequence of appraisal emotion, service quality, perceived value and customer satisfaction on repurchase intent in the performing arts. Journal of Services Marketing 24 (2): 170-182.

Hyun SS, Kim W et Lee MJ (2011) The impact of advertising on patrons' emotional responses, perceived value, and behavioral intentions in the chain restaurant industry: the moderating role of advertising-induced arousal. International Journal of Hospitality Management 30 (3): 689-700.

Izard CE (1977) Human Emotions. New York: Plenum.

Jones MA, Reynolds KE et Arnold MJ (2006) Hedonic and utilitarian shopping value: investigating differential effects on retail outcomes. Journal of Business Research 59 (9): 974981.

Jöreskog K (1971) Statistical analysis of sets of congeneric tests. Psychometrica 36 (2): 109133.

Kim M, Vogt CA et Knutson BJ (2013) Relationships among customer satisfaction, delight, and loyalty in the hospitality industry. Journal of Hospitality \& Tourism Research. Epub ahead of print 2 January 2013. DOI: 10.1177/1096348012471376.

Kreziak D et Frochot I (2011) Co-construction de l'expérience touristique: les stratégies des touristes en stations de sport d’hiver. Décisions marketing 64 (octobre-décembre): 23-33.

Kumar A, Olshavsky RW et King MF (2001) Exploring alternative antecedents of customer delight. Journal of Consumer Satisfaction Dissatisfaction and Complaining Behavior 14 1426.

Kwortnik RJ Jr et Ross WT Jr (2007) The role of positive emotions in experiential decisions. International Journal of Research in Marketing 24 (4): 324-335.

Ladhari R (2007) The effect of consumption emotions on satisfaction and word-of-mouth communications. Psychology \& Marketing 24 (12): 1085-1108.

Laros FJ et Steenkamp JBE (2005) Emotions in consumer behavior: a hierarchical approach. Journal of Business Research 58 (10): 1437-1445. 
Lee YK, Lee CK, Lee SK et Babin BJ (2008) Festivalscapes and patrons' emotions, satisfaction, and loyalty. Journal of Business Research 61 (1): 56-64.

Loureiro SM (2010) Satisfying and delighting the rural tourists. Journal of Travel \& Tourism Marketing 27 (4): 396-408.

Loureiro SMC et Kastenholz E (2011) Corporate reputation, satisfaction, delight, and loyalty towards rural lodging units in Portugal. International Journal of Hospitality Management 30 (3): 575-583.

McGinnis L, Gentry JW et Gao T (2008) The impact of flow and communitas on enduring involvement in extended service encounters. Journal of Service Research 11 (1): 74-90.

Magnini VP, Crotts JC et Zehrer A (2011) Understanding customer delight: an application of travel blog analysis. Journal of Travel Research 50 (5): 535-545.

Mano H et Oliver RL (1993) Assessing the dimensionality and structure of the consumption experience: evaluation, feeling and satisfaction. Journal of Consumer Research 20 (3): 451466.

Mathwick C, Malhotra N et Rigdon E (2001): Experiential value: conceptualization, measurement and application in the catalog and Internet shopping environment. Journal of Retailing 77 (1): 39-56.

Maubisson L (2012) L’immersion du consommateur dans une expérience de shopping. In: Actes du $28^{\text {ème }}$ congrès international de l'Association Française du Marketing, Brest, France, CD-Rom.

Mehrabian A et Russell JA (1974) An approach to environmental psychology. Cambridge: The MIT Press.

Oliver RL (1999) Value as excellence in the consumption experience. In Holbrook M (coord.) Consumer value: a framework for analysis and research. London and New York: Routledge Interpretive Market Research Series, pp.43-62.

Oliver RL, Rust RT et Varki S (1997) Customer delight: foundations, findings, and managerial insight. Journal of Retailing 73 (3): 311-336. 
Pine BJ et Gilmore JH (1998) Welcome to the experience economy. Harvard Business Review 76 (4): 97-105.

Plutchik R (1980) Emotion: a psycho-evolutionary synthesis. New York: Harper \& Row.

Plutchik R et Kellerman H (1974) Manual for the Emotions Profile Index. Los Angeles: Western Psychological Services.

Remy E (2007) Organisation et souffrance chez les hommes qui font les courses ! : Une approche à partir de la notion d'expérience de consommation ordinaire. In : 12èmes journées de recherche en marketing de Bourgogne, Dijon, France, 6, pp.59-71.

Richins ML (1997) Measuring emotions in the consumption experience. Journal of Consumer Research 24 (2): 127-146.

Ritzer G (2005) Enchanting a disenchanted world: revolutionizing the means of consumption. Thousand Oaks: Pine Forge Press.

Roederer C (2012) Contribution à la conceptualisation de l'expérience de consommation: émergence des dimensions de l'expérience au travers de récits de vie. Recherche et Applications en Marketing 27 (3): 81-96.

Rust RT et Oliver RL (2000) Should we delight the customer ? Journal of the Academy of Marketing Science 28 (1): 86-94.

Ruth JA, Brunel FF et Otnes CC (2002) Linking thoughts to feelings: investigating cognitive appraisals and consumption emotions in a mixed-emotions context. Journal of the Academy of Marketing Science 30 (1): 44-58.

St-James Y et Taylor S (2004) Delight-as-magic: refining the conceptual domain of customer delight. Advances in Consumer Research 31: 753-758.

Sanchez-Fernandez R, Iniesta-Bonillo AM et Holbrook MB (2009) The conceptualization and measurement of customer value in services. International Journal of Marketing Research 15 (1): 93-113.

Sands S, Oppewal H et Beverland M (2009) The effects of in-store themed events on consumer store choice decisions. Journal of Retailing and Consumer Services 16 (5): 386395. 
Schmitt B (1999) Experiential marketing. Journal of Marketing Management 15 (1-3): 53-67.

Shaw G, Bailey A et Williams A (2011) Aspects of service-dominant logic and its implications for tourism management: examples from the hotel industry. Tourism Management 32 (2): 207-214.

Sweeney JC et Soutar GN (2001) Consumer perceived value: the development of a multiple item scale. Journal of Retailing 77 (2): 203-220.

Tenenhaus M, Esposito Vinzi V, Chatelin YM et Lauro C (2005) PLS path modeling. Computational Statistics Data Analysis 48 (1): 159-205.

Torres EN et Kline S (2006) From satisfaction to delight: a model for the hotel industry. International Journal of Contemporary Hospitality Management 18 (4): 290-301.

Tsaur S, Yen C et Hsiao S (2013) Transcendent experience, flow and happiness for mountain climbers. International Journal of Tourism Research 15 (4): 360-374.

Vanhamme J (2002) La satisfaction des consommateurs spécifique à une transaction: définition, antécédents, mesures et modes. Recherche et Applications en Marketing 17 (2): 5585.

Vanhamme J (2008) La relation surprise-ravissement revisitée à l'aune du marketing expérientiel. Recherche et Applications en Marketing 23 (3): 115-141.

Vilares MJ, Almeida MH et Coelho PS (2010) Comparison of likelihood and PLS estimators for structural equation modeling: a simulation with customer satisfaction data. In Esposito Vinzi V, Chin WW, Henseler J et Wang H (coord.) Handbook of Partial Least Squares, New York: Springer Handbooks of Computational Statistics, pp.289-305.

Walsh G, Shiu E et Hassan LM (2014) Replicating, validating, and reducing the length of the consumer perceived value scale. Journal of Business Research 67 (3): 260-267.

Wirtz J et Bateson JE (1999) Consumer satisfaction with services: integrating the environment perspective in services marketing into the traditional disconfirmation paradigm. Journal of Business Research 44 (1): 55-66. 
Wirtz J, Mattila AS et Tan RL (2000) The moderating role of target-arousal on the impact of affect on satisfaction-an examination in the context of service experiences. Journal of Retailing 76 (3): 347-365.

Yau OHM et Kwong KK (2007) Searching for differences between delighted and satisfied customers: a structural equation model approach. In: 13 ème Asia Pacific management conference, Melbourne, Australie, pp.905-911.

Yi Y et Hoseong J (2003) Effects of loyalty programs on value perception, program loyalty and brand loyalty. Journal of the Academy of Marketing Science 31 (3): 229-240.

Yoon YS, Lee JS et Lee CK (2010) Measuring festival quality and value affecting visitors' satisfaction and loyalty using a structural approach. International Journal of Hospitality Management 29 (2): 335-342.

Zhao X, Lynch JG Jr et Chen Q (2011) Reconsidérer Baron et Kenny: mythes et vérités à propos de l'analyse de médiation. Recherche et Applications en Marketing 26 (1): 81-95. 
A1 : Structure et homogénéité des échantillons Arc 1800 et Arc 1950

\begin{tabular}{|c|c|c|c|c|c|}
\hline \multicolumn{2}{|c|}{ Caractéristiques } & & Arc & Test statistique & Interprétation \\
\hline \multirow{4}{*}{ Revenu } & Moins de 1200 euros & $2,8 \%$ & $2,6 \%$ & \multirow{4}{*}{$\begin{aligned} \mathrm{Chi}^{2} & =21,032 \\
\text { Sig } & =0,000\end{aligned}$} & \multirow{4}{*}{$\begin{array}{l}\text { Significatif - Les } \\
\text { touristes à fort } \\
\text { revenus sont } \\
\text { surreprésentés dans } \\
\text { la station Arc } 1950\end{array}$} \\
\hline & De 1200 à 2000 euros & $8 \%$ & $1,7 \%$ & & \\
\hline & De 2000 à 4000 euros & $28,6 \%$ & $18,3 \%$ & & \\
\hline & Plus de 4000 euros & $60 \%$ & $77,4 \%$ & & \\
\hline \multirow{2}{*}{ Genre } & Homme & $46,1 \%$ & $50,4 \%$ & \multirow{2}{*}{$\begin{array}{c}\mathrm{Chi}^{2}=0,984 \\
\text { Sig }=0,321\end{array}$} & \multirow{2}{*}{ Non significatif } \\
\hline & Femme & $53,9 \%$ & $49,6 \%$ & & \\
\hline \multirow{5}{*}{ Niveau d'étude } & Primaire & $100 \%$ & $0 \%$ & \multirow{5}{*}{$\begin{array}{l}\text { Chi }^{2}=7,927 \\
\text { Sig }=0,094\end{array}$} & \multirow{5}{*}{ Non significatif } \\
\hline & Collège / Lycée & $56,7 \%$ & $43,3 \%$ & & \\
\hline & $\mathrm{Bac}$ à Bac+2 & $60,6 \%$ & $39,4 \%$ & & \\
\hline & $\mathrm{Bac}+3 / 4$ & $54,5 \%$ & $45,5 \%$ & & \\
\hline & Bac +5 et plus & $51,2 \%$ & $48,8 \%$ & & \\
\hline \multirow{9}{*}{$\begin{array}{c}\text { Catégorie } \\
\text { socioprofessionnelle }\end{array}$} & Agriculteur & $50 \%$ & $50 \%$ & \multirow{9}{*}{$\begin{array}{l}\mathrm{Chi}^{2}=7,880 \\
\text { Sig }=0,445\end{array}$} & \multirow{9}{*}{ Non significatif } \\
\hline & $\begin{array}{l}\text { Commerçant, artisan, chef } \\
\text { d'entreprise }\end{array}$ & $51,8 \%$ & $48,2 \%$ & & \\
\hline & $\begin{array}{l}\text { Cadre, Profession } \\
\text { intellectuelle supérieure }\end{array}$ & $52,2 \%$ & $47,8 \%$ & & \\
\hline & Profession intermédiaire & $50 \%$ & $50 \%$ & & \\
\hline & Employé & $66,1 \%$ & $33,9 \%$ & & \\
\hline & Ouvrier & $100 \%$ & $0 \%$ & & \\
\hline & Chômeur & $66,7 \%$ & $33,3 \%$ & & \\
\hline & Elève, Etudiant & $61,7 \%$ & $38,3 \%$ & & \\
\hline & Inactif & $66,7 \%$ & $33,3 \%$ & & \\
\hline \multirow{2}{*}{$\begin{array}{l}\text { Implication durable } \\
\text { à l'égard des séjours } \\
\text { en stations }\end{array}$} & Score moyen & 2,4 & 2,3 & \multirow{2}{*}{$\begin{array}{c}t=1,086 \\
\text { Sig }=0,287\end{array}$} & \multirow{2}{*}{ Non significatif } \\
\hline & Ecart-type & 0,94 & 0,90 & & \\
\hline \multirow{2}{*}{$\begin{array}{l}\text { Niveau Optimal de } \\
\text { Stimulation }\end{array}$} & Score moyen & 3,99 & 4 & \multirow{2}{*}{$\begin{array}{c}t=-0,106 \\
\text { Sig }=0,916\end{array}$} & \multirow{2}{*}{ Non significatif } \\
\hline & Ecart-type & 0,96 & 0,99 & & \\
\hline
\end{tabular}


A2 : Adaptation des échelles de mesure mobilisées au contexte des séjours en stations de sports d'hiver - Etudes pilotes

\section{Adaptation du Consumption Emotions Set (CES)}

Le CES développé par Richins (1997) compte 43 émotions. Afin de l’adapter au contexte des séjours en stations de sports d'hiver, l'échelle de mesure a tout d'abord été soumise à un test de pertinence auprès d'un échantillon de convenance. Chaque individu interrogé devait apprécier la pertinence des 43 émotions recensées par le CES dans le cadre d’un séjour en station de ski $(1$ = absolument pas pertinent à 7 = extrêmement pertinent). Sur 267 individus interrogés, 137 réponses ont été retenues. Seules les réponses d’individus ayant déjà séjourné au minimum trois jours consécutifs en station de ski ont été conservées. Un regroupement des modalités de réponses a été effectué. Les émotions obtenant un score supérieur ou égal à 50\% sur le regroupement « pertinent » (modalités de 5 à 7 = émotion pertinente) ont été retenues. Cette procédure a conduit à conserver 12 émotions.

Par la suite, une analyse factorielle exploratoire a conduit à retenir une solution factorielle en trois dimensions (stimulation, joie, tranquillité) et 7 items. Les items présents sur deux dimensions, et dont les poids factoriels étaient supérieurs à 0,40 sur deux dimensions, ont été supprimés. De la même manière, les items dont les poids factoriels étaient inférieurs à 0,50 ont été éliminés (Hair et al, 1998).

\section{Développement d'une mesure de la valeur de consommation}

Afin de développer une mesure de la valeur de consommation adaptée au contexte des séjours en stations de sports d'hiver, soixante entretiens individuels semi directifs ont tout d'abord été réalisés auprès de clients de diverses stations des Alpes. Le corpus textuel a fait l'objet d'une analyse thématique manuelle afin de faire émerger les différentes sources de valeurs liées à ce type d'expérience spécifique. Par la suite, une liste de 52 items a été soumise à un échantillon de convenance de 456 individus et une analyse factorielle exploratoire a conduit à retenir 40 items se regroupant autour de 10 dimensions en adéquation avec les travaux antérieurs autour du concept de valeur de consommation. Les items présents sur deux dimensions, et dont les poids factoriels étaient supérieurs à 0,40 sur deux dimensions, ont été supprimés. De la même manière, les items dont les poids factoriels étaient inférieurs à 0,50 ont été éliminés (Hair et al, 1998). 
A3 : Construits, énoncés et loadings - Analyse Factorielle Confirmatoire

\begin{tabular}{|c|c|c|c|c|c|}
\hline \multirow{2}{*}{$\begin{array}{l}\text { Facteurs de } \\
\text { premier ordre }\end{array}$} & \multirow{2}{*}{ Items } & \multicolumn{2}{|c|}{ Arc 1800} & \multicolumn{2}{|c|}{ Arc 1950} \\
\hline & & Loadings & $t$ & Loadings & $t$ \\
\hline \multirow{2}{*}{ Stimulation } & Enthousiaste & 0,926 & $22,833 *$ & 0,906 & $17,073 *$ \\
\hline & Stimulé & 0,913 & $24,171 *$ & 0,908 & $18,407 *$ \\
\hline \multirow{3}{*}{ Joie } & Heureux & 0,930 & $20,635 *$ & 0,942 & $16,419 *$ \\
\hline & Content & 0,899 & $21,590 *$ & 0,926 & $19,020 *$ \\
\hline & Joyeux & 0,877 & $20,285 *$ & 0,898 & $13,256^{*}$ \\
\hline \multirow{2}{*}{ Tranquillité } & Calme & 0,920 & $19,480 *$ & 0,898 & $16,684^{*}$ \\
\hline & Paisible & 0,906 & $18,465 *$ & 0,886 & $14,457 *$ \\
\hline \multirow{3}{*}{ Enchantement } & Globalement, je peux dire que j’ai été enchanté par ce séjour & 0,774 & $14,565 *$ & 0,736 & $10,674 *$ \\
\hline & Globalement, je peux dire que ce séjour m’a émerveillé & 0,817 & $18,728 *$ & 0,850 & $16,615 *$ \\
\hline & Globalement, je peux dire que ce séjour m’a transporté & 0,835 & $18,245 *$ & 0,810 & $11,685^{*}$ \\
\hline \multirow{2}{*}{ Satisfaction } & Globalement, je suis satisfait de mon séjour & 0,956 & $23,295 *$ & 0,938 & $13,390 *$ \\
\hline & $\begin{array}{l}\text { Je trouve que le séjour a été à globalement la hauteur de mes } \\
\text { attentes }\end{array}$ & 0,972 & $26,605^{*}$ & 0,961 & $14,443^{*}$ \\
\hline \multirow{4}{*}{$\begin{array}{c}\text { Valeur } \\
\text { d'évasion }\end{array}$} & J'aime le sentiment de liberté que me procure la montagne & 0,725 & $10,668 *$ & 0,756 & $11,733 *$ \\
\hline & Pendant mon séjour en station je m'évade, j'oublie le quotidien & 0,731 & $12,008^{*}$ & 0,810 & $13,735 *$ \\
\hline & J'aime passer un séjour au ski car c'est un vrai dépaysement & 0,737 & $11,627^{*}$ & 0,746 & $12,338 *$ \\
\hline & $\begin{array}{l}\text { J'apprécie de venir en station parce que je découvre toujours } \\
\text { de nouveaux paysages, de nouvelles expériences, de nouvelles } \\
\text { personnes }\end{array}$ & 0,717 & $8,416^{*}$ & 0,701 & $7,325^{*}$ \\
\hline \multirow{5}{*}{$\begin{array}{c}\text { Valeur } \\
\text { d'esthétisme }\end{array}$} & $\begin{array}{l}\text { Les vacances au ski sont l'occasion de passer du temps dans } \\
\text { un bel endroit, de voir de belles choses }\end{array}$ & 0,835 & $12,395^{*}$ & 0,788 & $10,613^{*}$ \\
\hline & $\begin{array}{l}\text { Ce qui me plaît dans les séjours en station, c'est la beauté du } \\
\text { lieu }\end{array}$ & 0,805 & $14,343^{*}$ & 0,806 & $14,092 *$ \\
\hline & La montagne est quelque chose de beau que j'aime contempler & 0,768 & $12,949 *$ & 0,771 & $12,148^{*}$ \\
\hline & $\begin{array}{l}\text { Les séjours en station me donnent l'occasion de voir de beaux } \\
\text { paysages, de belles architectures }\end{array}$ & 0,780 & $9,363 *$ & 0,792 & $7,581^{*}$ \\
\hline & $\begin{array}{l}\text { En station, j'aime être dans un cadre esthétique, pour le plaisir } \\
\text { des yeux }\end{array}$ & 0,742 & $11,021^{*}$ & 0,779 & $11,734 *$ \\
\hline \multirow{5}{*}{$\begin{array}{l}\text { Valeur de } \\
\text { stimulation } \\
\text { expérientielle }\end{array}$} & $\begin{array}{l}\text { J'apprécie les séjours en station parce qu'ils sont l'occasion de } \\
\text { pratiquer beaucoup d'activités }\end{array}$ & 0,772 & $13,019 *$ & 0,803 & $13,013 *$ \\
\hline & J'aime être en station car c'est synonyme de loisirs divers & 0,787 & $12,042 *$ & 0,847 & $12,308^{*}$ \\
\hline & $\begin{array}{l}\text { J'apprécie de pouvoir vivre de nombreuses expériences } \\
\text { différentes lors de mes séjours au ski }\end{array}$ & 0,714 & 9,788* & 0,721 & 9,915* \\
\hline & $\begin{array}{l}\text { Les séjours en station sont l'occasion de connaître de } \\
\text { nouvelles choses }\end{array}$ & 0,749 & $13,562 *$ & 0,802 & $13,875^{*}$ \\
\hline & $\begin{array}{l}\text { J'aime apprendre à pratiquer de nouvelles activités lors de mes } \\
\text { séjours }\end{array}$ & 0,625 & 7,367* & 0,742 & $8,717^{*}$ \\
\hline
\end{tabular}




\begin{tabular}{|c|c|c|c|c|c|}
\hline \multirow{4}{*}{$\begin{array}{l}\text { Valeur } \\
\text { hédonique }\end{array}$} & Les vacances au ski sont synonyme de "bien vivre" & 0,814 & $12,754 *$ & 0,857 & $13,723 *$ \\
\hline & $\begin{array}{l}\text { J'aime les séjours au ski parce qu'ils me permettent de profiter } \\
\text { de la vie }\end{array}$ & 0,828 & $11,965 *$ & 0,784 & $9,450 *$ \\
\hline & En station, je profite du soleil, de la neige, de la nature & 0,596 & $7,442 *$ & 0,717 & $8,886^{*}$ \\
\hline & $\begin{array}{l}\text { Lors de mes séjours en station, j'aime bien manger, profiter de } \\
\text { la cuisine locale }\end{array}$ & 0,616 & $8,063^{*}$ & 0,731 & $8,859 *$ \\
\hline \multirow{3}{*}{$\begin{array}{l}\text { Valeur de } \\
\text { pratique sociale }\end{array}$} & $\begin{array}{l}\text { Les séjours au ski, c'est pouvoir partager une expérience avec } \\
\text { sa famille ou ses amis }\end{array}$ & 0,567 & $6,418^{*}$ & 0,678 & $4,865 *$ \\
\hline & $\begin{array}{l}\text { Je conçois les vacances au ski comme l'occasion de se } \\
\text { retrouver, d'être ensemble }\end{array}$ & 0,667 & $8,740^{*}$ & 0,733 & $5,838^{*}$ \\
\hline & $\begin{array}{l}\text { Ce qui me plaît en station, c'est qu'il y a de quoi faire pour } \\
\text { tout le monde, c'est du bonheur pour tous }\end{array}$ & 0,925 & $14,037 *$ & 0,916 & $10,279 *$ \\
\hline \multirow{3}{*}{$\begin{array}{l}\text { Valeur de } \\
\text { communion } \\
\text { Sociale }\end{array}$} & $\begin{array}{l}\text { Quand je suis en station, j'ai l'impression de partager les } \\
\text { mêmes émotions avec les autres personnes en vacances }\end{array}$ & 0,728 & $14,366^{*}$ & 0,824 & $16,211^{*}$ \\
\hline & $\begin{array}{l}\text { Il y a un lien qui unit les personnes qui sont en vacances au } \\
\text { ski }\end{array}$ & 0,863 & $17,436^{*}$ & 0,877 & $21,003^{*}$ \\
\hline & $\begin{array}{l}\text { Lors de mes séjours en station, c'est comme si je faisais partie } \\
\text { d'une sorte de "communauté" qui ressent des émotions } \\
\text { collectivement }\end{array}$ & 0,820 & $17,701^{*}$ & 0,879 & $21,009 *$ \\
\hline \multirow{4}{*}{$\begin{array}{l}\text { Valeur } \\
\text { d'interaction } \\
\text { sociale }\end{array}$} & $\begin{array}{l}\text { J'apprécie de pouvoir rencontrer de nouvelles personnes lors } \\
\text { de mes séjours en station }\end{array}$ & 0,871 & $18,034 *$ & 0,895 & $19,165^{*}$ \\
\hline & $\begin{array}{l}\text { Les séjours au ski sont un moyen de rencontrer la population } \\
\text { locale }\end{array}$ & 0,562 & $8,730^{*}$ & 0,770 & $15,012^{*}$ \\
\hline & $\begin{array}{l}\text { Pendant mon séjour, je peux créer des contacts divers et variés } \\
\text { facilement }\end{array}$ & 0,817 & $18,211^{*}$ & 0,787 & $13,808^{*}$ \\
\hline & $\begin{array}{l}\text { J'apprécie la possibilité d'avoir des échanges, de discuter avec } \\
\text { des personnes diverses lors de mes séjours }\end{array}$ & 0,845 & $18,170^{*}$ & 0,844 & $16,164 *$ \\
\hline \multirow{4}{*}{$\begin{array}{l}\text { Valeur } \\
\text { d'expression de } \\
\text { soi }\end{array}$} & $\begin{array}{l}\text { Ma personnalité compte beaucoup dans le choix d'un séjour } \\
\text { au ski ou dans le choix de la station }\end{array}$ & 0,752 & $12,610^{*}$ & 0,813 & $13,737^{*}$ \\
\hline & $\begin{array}{l}\text { J'ai l'impression d'exprimer qui je suis lors de mes vacances } \\
\text { au ski }\end{array}$ & 0,742 & $14,436^{*}$ & 0,728 & $11,283^{*}$ \\
\hline & $\begin{array}{l}\text { Je pense que je choisis en partie la station où je vais en } \\
\text { fonction de ma personnalité }\end{array}$ & 0,794 & $14,717^{*}$ & 0,782 & $11,646^{*}$ \\
\hline & $\begin{array}{l}\text { Le fait de partir en vacances au ski me permet d'exprimer qui } \\
\text { je suis }\end{array}$ & 0,825 & $17,475^{*}$ & 0,773 & $12,569 *$ \\
\hline \multirow{3}{*}{$\begin{array}{l}\text { Valeur de } \\
\text { distinction } \\
\text { sociale }\end{array}$} & $\begin{array}{l}\text { Les vacances au ski sont réservées à une certaine catégorie de } \\
\text { personnes }\end{array}$ & 0,771 & $8,173^{*}$ & 0,758 & 9,537* \\
\hline & $\begin{array}{l}\text { Le fait de pouvoir passer un séjour en station me fait me sentir } \\
\text { privilégié }\end{array}$ & 0,897 & $12,502 *$ & 0,866 & $9,574^{*}$ \\
\hline & $\begin{array}{l}\text { Tout le monde ne peut pas s'offrir un séjour en station, et je } \\
\text { suis content de pouvoir le faire }\end{array}$ & 0,665 & $7,399 *$ & 0,751 & $9,807^{*}$ \\
\hline \multirow{5}{*}{$\begin{array}{c}\text { Valeur } \\
\text { fonctionnelle }\end{array}$} & J'aime quand tout est accessible et pratique dans une station & 0,795 & $8,742^{*}$ & 0,844 & $8,288^{*}$ \\
\hline & J'apprécie que les pistes soient bien entretenues et balisées & 0,770 & $8,203^{*}$ & 0,853 & $8,404 *$ \\
\hline & $\begin{array}{l}\text { La qualité ou l'emplacement du logement sont importantes } \\
\text { pour moi }\end{array}$ & 0,771 & $11,673^{*}$ & 0,836 & $10,577^{*}$ \\
\hline & $\begin{array}{l}\text { Une météo et/ou un enneigement favorables sont essentiels } \\
\text { pour passer un bon séjour }\end{array}$ & 0,673 & $7,411^{*}$ & 0,806 & $10,499 *$ \\
\hline & $\begin{array}{l}\text { J'apprécie que la station propose de nombreux services } \\
\text { (Ecoles de ski, bars, restaurants, supermarchés, boutiques, } \\
\text { jardins d'enfants, laverie, activités sportives, animations, etc.) }\end{array}$ & 0,734 & $8,950^{*}$ & 0,816 & $8,593^{*}$ \\
\hline
\end{tabular}

* Coefficient significatif. Les valeurs du test en $t$ de Student supérieures à $|2,575|$ indiquent des paramètres significatifs au seuil de 1 $\%$. 
A4 : Liens établis entre les facteurs de premier et de second ordre

\begin{tabular}{|c|c|c|c|c|}
\hline \multirow{2}{*}{ Facteur de premier ordre $\leftarrow$ Facteur de second ordre } & \multicolumn{2}{|c|}{ Arc 1800} & \multicolumn{2}{|c|}{ Arc 1950} \\
\hline & $\begin{array}{c}\text { Path } \\
\text { coefficients }\end{array}$ & $t$ & $\begin{array}{c}\text { Path } \\
\text { coefficients }\end{array}$ & $t$ \\
\hline Stimulation $\leftarrow$ Emotions élémentaires positives & 0,872 & $30,557^{*}$ & 0,844 & $24,466^{*}$ \\
\hline Joie $\leftarrow$ Emotions élémentaires positives & 0,929 & $43,005 *$ & 0,913 & $34,928 *$ \\
\hline Tranquillité — Emotions élémentaires positives & 0,804 & $23,263^{*}$ & 0,797 & $20,529 *$ \\
\hline Valeur d'évasion $\leftarrow$ Valeur plaisir & 0,837 & $26,236 *$ & 0,859 & $26,056 *$ \\
\hline Valeur d’esthétisme $\leftarrow$ Valeur plaisir & 0,817 & $24,349 *$ & 0,791 & $20,145^{*}$ \\
\hline Valeur de stimulation expérientielle $\leftarrow$ Valeur plaisir & 0,779 & $21,322 *$ & 0,756 & $17,945^{*}$ \\
\hline Valeur hédonique $\leftarrow$ Valeur plaisir & 0,813 & $23,995 *$ & 0,862 & $26,435 *$ \\
\hline Valeur de pratique sociale $\leftarrow$ Valeur sociale & 0,620 & $13,558 *$ & 0,566 & $10,690 *$ \\
\hline Valeur de communion sociale $\leftarrow$ Valeur sociale & 0,887 & $32,949 *$ & 0,921 & $36,864^{*}$ \\
\hline Valeur d'interaction sociale $\leftarrow$ Valeur sociale & 0,887 & $32,971 *$ & 0,900 & $32,067 *$ \\
\hline Valeur d'expression de soi $\leftarrow$ Valeur de distinction & 0,911 & $38,022 *$ & 0,864 & $26,751^{*}$ \\
\hline Valeur de distinction sociale $\leftarrow$ Valeur de distinction & 0,643 & $14,092 *$ & 0,752 & $17,726^{*}$ \\
\hline
\end{tabular}

* Coefficient significatif. Les valeurs du test en $t$ de Student supérieures à $|2,575|$ indiquent des paramètres significatifs au seuil de $1 \%$. 
A5 : Coefficients de fiabilité

\begin{tabular}{|l|c|c|c|c|}
\hline \multirow{2}{*}{} & \multicolumn{2}{|c|}{ Alpha de Cronbach } & \multicolumn{2}{c|}{ Rhô de Jöreskog } \\
\cline { 2 - 5 } & Arc 1800 & Arc 1950 & \multirow{2}{*}{ Arc 1800 } & \multirow{2}{*}{ Arc 1950} \\
\hline Emotions élémentaires positives* & 0,904 & 0,895 & 0,971 & 0,971 \\
\hline Stimulation & 0,817 & 0,784 & 0,916 & 0,903 \\
\hline Joie & 0,886 & 0,912 & 0,929 & 0,945 \\
\hline Tranquillité & 0,801 & 0,744 & 0,910 & 0,887 \\
\hline Enchantement & 0,742 & 0,733 & 0,852 & 0,845 \\
\hline Satisfaction & 0,925 & 0,892 & 0,964 & 0,949 \\
\hline Valeur de plaisir* & 0,896 & 0,910 & 0,957 & 0,965 \\
\hline Valeur d'évasion & 0,716 & 0,746 & 0,816 & 0,840 \\
\hline Valeur d'esthétisme & 0,848 & 0,848 & 0,890 & 0,890 \\
\hline Valeur de stimulation expérientielle & 0,784 & 0,844 & 0,853 & 0,889 \\
\hline Valeur hédonique & 0,693 & 0,778 & 0,814 & 0,858 \\
\hline Valeur sociale* & 0,831 & 0,859 & 0,937 & 0,954 \\
\hline Valeur de pratique sociale & 0,677 & 0,735 & 0,816 & 0,848 \\
\hline Valeur de communion sociale & 0,728 & 0,824 & 0,848 & 0,895 \\
\hline Valeur d'interaction sociale & 0,781 & 0,843 & 0,861 & 0,895 \\
\hline Valeur de distinction* & 0,735 & 0,754 & 0,916 & 0,917 \\
\hline Valeur de distinction de soi & 0,786 & 0,781 & 0,860 & 0,858 \\
\hline Valeur de distinction sociale & 0,699 & 0,712 & 0,837 & 0,838 \\
\hline Valeur fonctionnelle & 0,805 & 0,888 & 0,864 & 0,918 \\
\hline
\end{tabular}

* Facteur de second ordre 


\section{A6 : Tests des validités convergentes et discriminantes}

\begin{tabular}{|c|c|c|c|c|c|c|c|c|c|c|c|c|c|c|c|c|c|c|c|c|}
\hline & & 1 & 2 & 3 & 4 & 5 & 6 & 7 & 8 & 9 & 10 & 11 & 12 & 13 & 14 & 15 & 16 & 17 & 18 & 19 \\
\hline \multirow{18}{*}{$\begin{array}{c}\text { Arc } \\
1800\end{array}$} & 1. Emotions élémentaires positives* & 0,829 & & & & & & & & & & & & & & & & & & \\
\hline & 2. Stimulation & & 0,845 & & & & & & & & & & & & & & & & & \\
\hline & 3. Joie & & 0,538 & 0,814 & & & & & & & & & & & & & & & & \\
\hline & 4. Tranquillité & & 0,283 & 0,407 & 0,834 & & & & & & & & & & & & & & & \\
\hline & 5. Enchantement* & 0,384 & 0,342 & 0,330 & 0,201 & 0,655 & & & & & & & & & & & & & & \\
\hline & 6. Satisfaction & 0,367 & 0,259 & 0,325 & 0,249 & 0,448 & 0,930 & & & & & & & & & & & & & \\
\hline & 7. Valeur de plaisir* & 0,225 & 0,241 & 0,200 & 0,080 & 0,211 & 0,132 & 0,554 & & & & & & & & & & & & \\
\hline & 8. Valeur d'évasion & 0,133 & 0,150 & 0,122 & 0,039 & 0,131 & 0,078 & & 0,529 & & & & & & & & & & & \\
\hline & 9. Valeur d'esthétisme & 0,138 & 0,125 & 0,147 & 0,045 & 0,094 & 0,087 & & 0,344 & 0,619 & & & & & & & & & & \\
\hline & 10. Valeur de stimulation expérientielle & 0,137 & 0,164 & 0,099 & 0,060 & 0,205 & 0,071 & & 0,325 & 0,158 & 0,535 & & & & & & & & & \\
\hline & 12. Valeur sociale* & 0,112 & 0,140 & 0,091 & 0,035 & 0,195 & 0,086 & 0,414 & 0,256 & 0,188 & 0,463 & 0,165 & 0,603 & & & & & & & \\
\hline & 13. Valeur de pratique sociale & 0,144 & 0,127 & 0,125 & 0,074 & 0,110 & 0,091 & 0,464 & 0,355 & 0,279 & 0,306 & 0,279 & & 0,541 & & & & & & \\
\hline & 14. Valeur de communion sociale & 0,055 & 0,076 & 0,046 & 0,011 & 0,153 & 0,048 & 0,238 & 0,151 & 0,108 & 0,259 & 0,099 & & 0,188 & 0,649 & & & & & \\
\hline & 15. Valeur d'interaction sociale & 0,078 & 0,108 & 0,059 & 0,023 & 0,135 & 0,060 & 0,273 & 0,144 & 0,102 & 0,403 & 0,078 & & 0,148 & 0,406 & 0,614 & & & & \\
\hline & 16. Valeur de distinction* & 0,054 & 0,075 & 0,038 & 0,018 & 0,076 & 0,017 & 0,156 & 0,056 & 0,113 & 0,112 & 0,132 & 0,160 & 0,080 & 0,141 & 0,103 & 0,610 & & & \\
\hline & 17. Valeur de distinction de soi & 0,039 & 0,071 & 0,027 & 0,006 & 0,093 & 0,021 & 0,104 & 0,037 & 0,044 & 0,115 & 0,080 & 0,159 & 0,042 & 0,153 & 0,115 & & 0,607 & & \\
\hline & 18. Valeur de distinction sociale & 0,030 & 0,020 & 0,022 & 0,027 & 0,006 & 0,001 & 0,102 & 0,038 & 0,154 & 0,022 & 0,103 & 0,037 & 0,079 & 0,022 & 0,014 & & 0,067 & 0,614 & \\
\hline & 19. Valeur Fonctionnelle* & 0,092 & 0,080 & 0,089 & 0,041 & 0,020 & 0,028 & 0,266 & 0,183 & 0,251 & 0,047 & 0,362 & 0,068 & 0,235 & 0,038 & 0,014 & 0,027 & 0,007 & 0,052 & 0,562 \\
\hline \multirow{17}{*}{$\begin{array}{c}\text { Arc } \\
1950\end{array}$} & 1. Emotions élémentaires positives* & 0,827 & & & & & & & & & & & & & & & & & & \\
\hline & 2. Stimulation & & 0,823 & & & & & & & & & & & & & & & & & \\
\hline & 5. Enchantement* & 0,435 & 0,424 & 0,347 & 0,190 & 0,640 & & & & & & & & & & & & & & \\
\hline & 6. Satisfaction & 0,445 & 0,296 & 0,438 & 0,233 & 0,402 & 0,901 & & & & & & & & & & & & & \\
\hline & 7. Valeur de plaisir* & 0,320 & 0,239 & 0,268 & 0,189 & 0,335 & 0,244 & 0,603 & & & & & & & & & & & & \\
\hline & 8. Valeur d'évasion & 0,228 & 0,190 & 0,175 & 0,135 & 0,238 & 0,200 & & 0,569 & & & & & & & & & & & \\
\hline & 9. Valeur d'esthétisme & 0,200 & 0,116 & 0,191 & 0,130 & 0,135 & 0,194 & & 0,394 & 0,620 & & & & & & & & & & \\
\hline & 10. Valeur de stimulation expérientielle & 0,176 & 0,154 & 0,135 & 0,096 & 0,334 & 0,093 & & 0,278 & 0,110 & 0,615 & & & & & & & & & \\
\hline & 11. Valeur hédonique & 0,255 & 0,181 & 0,225 & 0,149 & 0,177 & 0,194 & & 0,469 & 0,471 & 0,246 & 0,600 & & & & & & & & \\
\hline & 12. Valeur sociale* & 0,171 & 0,183 & 0,111 & 0,091 & 0,358 & 0,114 & 0,404 & 0,216 & 0,119 & 0,557 & 0,203 & 0,678 & & & & & & & \\
\hline & 13. Valeur de pratique sociale & 0,261 & 0,176 & 0,226 & 0,167 & 0,206 & 0,190 & 0,567 & 0,480 & 0,316 & 0,290 & 0,456 & & 0,612 & & & & & & \\
\hline & 14. Valeur de communion sociale & 0,128 & 0,159 & 0,067 & 0,070 & 0,302 & 0,077 & 0,267 & 0,130 & 0,078 & 0,397 & 0,126 & & 0,185 & 0,740 & & & & & \\
\hline & 15. Valeur d'interaction sociale & 0,080 & 0,095 & 0,052 & 0,035 & 0,244 & 0,055 & 0,230 & 0,096 & 0,039 & 0,467 & 0,086 & & 0,106 & 0,510 & 0,682 & & & & \\
\hline & 16. Valeur de distinction* & 0,190 & 0,207 & 0,117 & 0,105 & 0,242 & 0,115 & 0,282 & 0,164 & 0,149 & 0,211 & 0,220 & 0,222 & 0,187 & 0,207 & 0,113 & 0,613 & & & \\
\hline & 17. Valeur de distinction de soi & 0,136 & 0,180 & 0,073 & 0,065 & 0,180 & 0,061 & 0,181 & 0,086 & 0,055 & 0,189 & 0,162 & 0,228 & 0,111 & 0,209 & 0,149 & & 0,600 & & \\
\hline & 18. Valeur de distinction sociale & 0,114 & 0,091 & 0,084 & 0,076 & 0,137 & 0,100 & 0,196 & 0,144 & 0,177 & 0,088 & 0,127 & 0,069 & 0,143 & 0,068 & 0,016 & & 0,101 & 0,629 & \\
\hline & 19. Valeur Fonctionnelle* & 0,111 & 0,033 & 0,127 & 0,095 & 0,048 & 0,159 & 0,355 & 0,243 & 0,394 & 0,050 & 0,438 & 0,049 & 0,295 & 0,019 & 0,009 & 0,063 & 0,016 & 0,093 & 0,691 \\
\hline
\end{tabular}

Sur les diagonales sont fournies les validités convergentes $\left(\rho_{v c}\right)$ et sous les diagonales, sont fournis les carrés de la corrélation $\left(R_{i j}^{2}\right)$.

* Facteur de second ordre 
A7 : Tests de médiation



* Coefficient significatif. Les valeurs du test en $t$ de Student supérieures à $|2,575|$ indiquent des paramètres significatifs au seuil de $1 \%$.

** Coefficient significatif. Les valeurs du test en $t$ de Student supérieures à |1,96| indiquent des paramètres significatifs au seuil de $5 \%$. n.s. signifie que le coefficient n'est pas significatif. 
Tableau 1 : Intérêts et limites associés aux concepts mobilisés pour appréhender les effets des stratégies expérientielles sur le consommateur

\begin{tabular}{|c|c|c|}
\hline & Principaux intérêts & Principales limites \\
\hline $\begin{array}{l}\text { Les émotions } \\
\text { élémentaires } \\
\text { positives }\end{array}$ & $\begin{array}{l}\text { - Les émotions positives élémentaires et discrètes } \\
\text { permettent de rendre compte de la variété et de la } \\
\text { complexité du vécu lors d’expériences de consommation } \\
\text { (Richins, } 1997 \text {; Derbaix et Filser, 2011). } \\
\text { - La supériorité des mesures d'émotions primaires } \\
\text { discrètes (notamment le Consumption Emotion Set) sur } \\
\text { d'autres instruments de mesure a été montrée dans le cas } \\
\text { d'expériences de consommation (Richins, 1997). }\end{array}$ & $\begin{array}{l}\text { - Les émotions recensées varient fortement en fonction } \\
\text { des contextes d'étude (Ferrandi, De Barnier et Valette- } \\
\text { Florence, } 2002 \text {; Laros et Steenkamp, 2005). } \\
\text { - La variété des émotions recensées engendre des } \\
\text { problèmes de compréhension des items de la part des } \\
\text { répondants (Graillot, 1998). }\end{array}$ \\
\hline $\begin{array}{l}\text { L’enchantement } \\
\text { comme émotion } \\
\text { positive } \\
\text { complexe }\end{array}$ & $\begin{array}{l}\text { - L’enchantement, définit comme une émotion positive, } \\
\text { permet de rendre compte des réponses émotionnelles } \\
\text { dans un contexte expérientiel à forte intensité } \\
\text { émotionnelle (St-James et Taylor, } 2004 \text {; Finn, 2005). }\end{array}$ & $\begin{array}{l}\text { - Les essences conceptuelles de l'enchantement défini } \\
\text { comme émotion peuvent varier en fonction de la nature } \\
\text { de l'expérience (St-James et Taylor, 2004). } \\
\text { - Des problèmes de validité discriminante entre } \\
\text { l'enchantement et les émotions primaires telles que la } \\
\text { surprise ou la joie subsistent (Oliver, Rust et Varki, } \\
1997 \text {; Vanhamme, 2008). }\end{array}$ \\
\hline $\mathrm{La}$ & $\begin{array}{l}\text { - La conceptualisation de la satisfaction avec une } \\
\text { dimension affective permet d'appréhender l'évaluation } \\
\text { d'une consommation intégrant des attributs symboliques } \\
\text { et/ou de nature affective (Mukhopadhyay, 2005). } \\
\text { - Les modèles de satisfaction apparaissent pour les } \\
\text { managers comme des outils parcimonieux, simples à } \\
\text { administrer afin d'appréhender les effets des offres } \\
\text { d'expérience sur le consommateur (Vanhamme, 2002). }\end{array}$ & $\begin{array}{l}\text { - La mesure de la satisfaction ne permet pas une } \\
\text { différenciation fine des niveaux positifs de satisfaction } \\
\text { éprouvés par le consommateur lors des expériences } \\
\text { vécues (Vanhamme, 2008). } \\
\text { - Les échelles de la satisfaction ne captent pas de façon } \\
\text { satisfaisante la dimension affective du concept (Oliver, } \\
1997 \text {; Finn, 2005). }\end{array}$ \\
\hline $\begin{array}{l}\text { La valeur de } \\
\text { consommation }\end{array}$ & $\begin{array}{l}\text { - L’approche factorielle de la valeur de consommation } \\
\text { offre un cadre d'analyse applicable à tout type } \\
\text { d'expérience vécue (Holbrook, 1999). } \\
\text { - La valeur de consommation recèle un potentiel } \\
\text { opérationnel élevé, notamment pour identifier des } \\
\text { leviers de création de valeur en lien avec l’expérience } \\
\text { vécue par le consommateur (Badot et Heilbrunn, 2006). }\end{array}$ & $\begin{array}{l}\text { - Le concept de valeur a fait l'objet d’opérationnalisations } \\
\text { variées (bénéfices, sacrifices ou dimensions de la valeur } \\
\text { perçues) qui rendent difficile la comparaison des résultats } \\
\text { obtenus (Walsh, Shiu et Hassan, 2014). } \\
\text { - Les instruments de mesure ne reflètent que } \\
\text { partiellement la richesse théorique du concept de valeur } \\
\text { de consommation (Sanchez-Fernandez, Iniesta-Bonillo et } \\
\text { Holbrook, 2009). }\end{array}$ \\
\hline
\end{tabular}


Tableau 2 : Résultats du modèle d'équations structurelles

\begin{tabular}{|c|c|c|c|c|c|c|c|}
\hline & \multicolumn{3}{|c|}{ Arc 1800} & \multicolumn{3}{|c|}{ Arc 1950} & \multirow{2}{*}{$\begin{array}{c}\text { Résultats (p-value) des } \\
\text { tests } \\
\text { de différences entre } \\
\text { les deux paramètres } \\
\text { (path coefficients) } \\
\text { réalisés }\end{array}$} \\
\hline & $\begin{array}{c}\text { Path } \\
\text { coefficients }\end{array}$ & $t$ & $\mathrm{R}^{2}$ & $\begin{array}{c}\text { Path } \\
\text { coefficients }\end{array}$ & $t$ & $\mathrm{R}^{2}$ & \\
\hline $\begin{array}{l}\text { Emotions élémentaires positives } \\
\rightarrow \text { Enchantement }\end{array}$ & 0,644 & $14,467^{*}$ & 0,415 & 0,678 & $14,349^{*}$ & 0,460 & 0,484 \\
\hline $\begin{array}{l}\text { Emotions élémentaires positives } \\
\rightarrow \text { Valeur plaisir }\end{array}$ & 0,296 & $4,555^{*}$ & \multirow{2}{*}{0,276} & 0,312 & $4,588^{*}$ & \multirow{2}{*}{0,396} & 0,874 \\
\hline Enchantement $\rightarrow$ Valeur plaisir & 0,284 & $4,371^{*}$ & & 0,374 & $5,499 *$ & & 0,353 \\
\hline $\begin{array}{l}\text { Emotions élémentaires positives } \rightarrow \\
\text { Valeur sociale }\end{array}$ & 0,089 & 1,311 (n.s.) & \multirow[t]{2}{*}{0,204} & 0,048 & 0,672 (n.s.) & \multirow[t]{2}{*}{0,347} & 0,694 \\
\hline Enchantement $\boldsymbol{\rightarrow}$ Valeur sociale & 0,389 & $5,716^{*}$ & & 0,556 & $7,844^{*}$ & & 0,105 \\
\hline $\begin{array}{l}\text { Emotions élémentaires positives } \rightarrow \\
\text { Valeur de distinction }\end{array}$ & 0,098 & 1,339 (n.s.) & \multirow{2}{*}{0,084} & 0,197 & $2,611 *$ & \multirow{2}{*}{0,260} & 0,389 \\
\hline $\begin{array}{llll}\begin{array}{l}\text { Enchantement } \\
\text { distinction }\end{array} & \rightarrow & \text { Valeur } & \text { de } \\
\end{array}$ & 0,217 & $2,973 *$ & & 0,356 & $4,724^{*}$ & & 0,243 \\
\hline $\begin{array}{l}\text { Emotions élémentaires positives } \\
\rightarrow \text { Valeur fonctionnelle }\end{array}$ & 0,354 & $4,887 *$ & \multirow{2}{*}{0,098} & 0,304 & $3,696 *$ & \multirow{2}{*}{0,119} & 0,695 \\
\hline $\begin{array}{lll}\begin{array}{l}\text { Enchantement } \\
\text { fonctionnelle }\end{array} & \rightarrow & \text { Valeur } \\
\end{array}$ & 0,072 & 1,000 (n.s.) & & 0,056 & 0,685 (n.s.) & & 0,252 \\
\hline $\begin{array}{l}\text { Emotions élémentaires positives } \\
\rightarrow \text { Satisfaction }\end{array}$ & 0,240 & $4,497 *$ & \multirow{6}{*}{0,563} & 0,330 & $5,540^{*}$ & \multirow{6}{*}{0,593} & 0,329 \\
\hline Enchantement $\rightarrow$ Satisfaction & 0,597 & $10,944^{*}$ & & 0,508 & $7,882^{*}$ & & 0,306 \\
\hline Valeur plaisir $\boldsymbol{\rightarrow}$ Satisfaction & 0,008 & 0,134 (n.s.) & & 0,031 & 0,415 (n.s.) & & 0,704 \\
\hline Valeur sociale $\rightarrow$ Satisfaction & 0,030 & 0,569 (n.s.) & & 0,110 & $1,838 * * *$ & & 0,334 \\
\hline $\begin{array}{lll}\text { Valeur de } & \text { distinction } & \rightarrow \\
\text { Satisfaction }\end{array}$ & 0,080 & $1,834 * * *$ & & 0,040 & 0,770 (n.s.) & & 0,588 \\
\hline $\begin{array}{lll}\text { Valeur fonctionnelle } & \rightarrow \\
\text { Satisfaction } & & \\
\end{array}$ & 0,018 & 0,393 (n.s.) & & 0,222 & $4,106^{*}$ & & 0,008 \\
\hline $\begin{array}{l}\text { Emotions élémentaires positives } \\
\rightarrow \text { Intentions comportementales }\end{array}$ & 0,040 & 0,607 (n.s.) & \multirow{7}{*}{0,367} & 0,032 & 0,410 (n.s.) & \multirow{7}{*}{0,383} & 0,413 \\
\hline $\begin{array}{l}\text { Enchantement } \rightarrow \\
\text { comportementales }\end{array}$ & 0,198 & $2,529 * *$ & & 0,242 & $2,706 *$ & & 0,719 \\
\hline $\begin{array}{l}\text { Valeur plaisir } \rightarrow \\
\text { comportementales }\end{array}$ & 0,112 & 1,498 (n.s.) & & 0,151 & 1,628 (n.s.) & & 0,739 \\
\hline $\begin{array}{l}\text { Valeur sociale } \rightarrow \text { Intentions } \\
\text { comportementales }\end{array}$ & 0,042 & 0,655 (n.s.) & & 0,001 & 0,015 (n.s.) & & 0,701 \\
\hline $\begin{array}{l}\text { Valeur de distinction } \rightarrow \text { Intentions } \\
\text { comportementales }\end{array}$ & 0,049 & 0,921 (n.s.) & & 0,064 & 1,017 (n.s.) & & 0,834 \\
\hline $\begin{array}{l}\text { Valeur fonctionnelle } \rightarrow \text { Intentions } \\
\text { comportementales }\end{array}$ & 0,086 & 1,536 (n.s.) & & 0,038 & 0,453 (n.s.) & & 0,188 \\
\hline $\begin{array}{lll}\begin{array}{l}\text { Satisfaction } \rightarrow \\
\text { comportementales }\end{array} & \text { Intentions } \\
& \end{array}$ & 0,456 & $6,442 *$ & & 0,532 & $6,641^{*}$ & & 0,491 \\
\hline GoF externe & \multicolumn{3}{|c|}{0,994} & \multicolumn{3}{|c|}{0,997} & \\
\hline GoF interne & \multicolumn{3}{|c|}{0,826} & \multicolumn{3}{|c|}{0,841} & \\
\hline
\end{tabular}

* Coefficient significatif. Les valeurs du test en $t$ de Student supérieures à |2,575| indiquent des paramètres significatifs au seuil de $1 \%$.

** Coefficient significatif. Les valeurs du test en $t$ de Student supérieures à $|1,96|$ indiquent des paramètres significatifs au seuil de $5 \%$.

*** Coefficient significatif. Les valeurs du test en $t$ de Student supérieures à $|1,645|$ indiquent des paramètres significatifs au seuil de $10 \%$.

(n.s.) Coefficient non-significatif. 
Figure 1 : Modèle intégrateur des effets générés sur le consommateur par un contexte expérientiel contrôlé

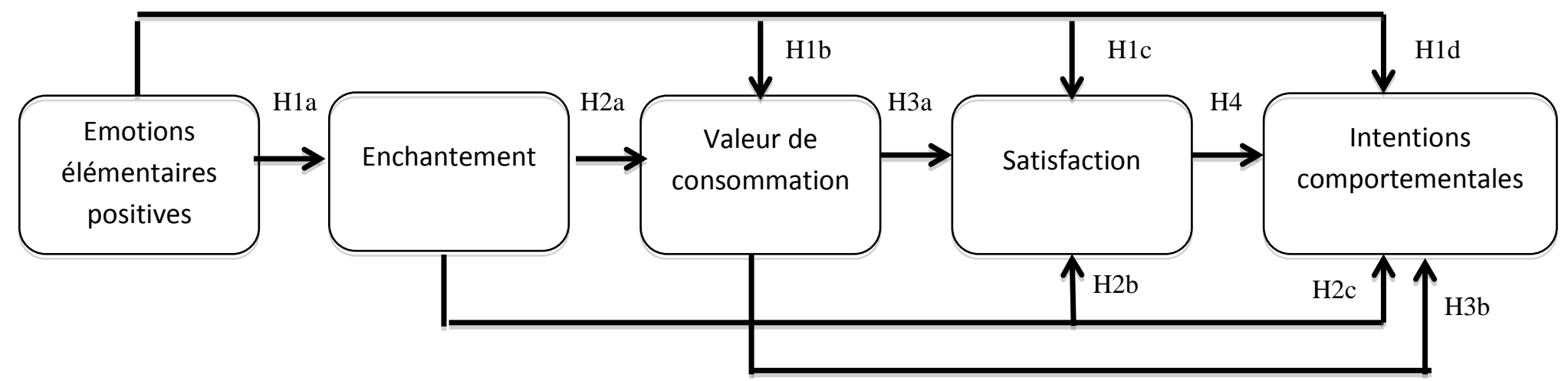


Figure 2 : Résultats du modèle d’équations structurelles - Arc 1800 (n=297)

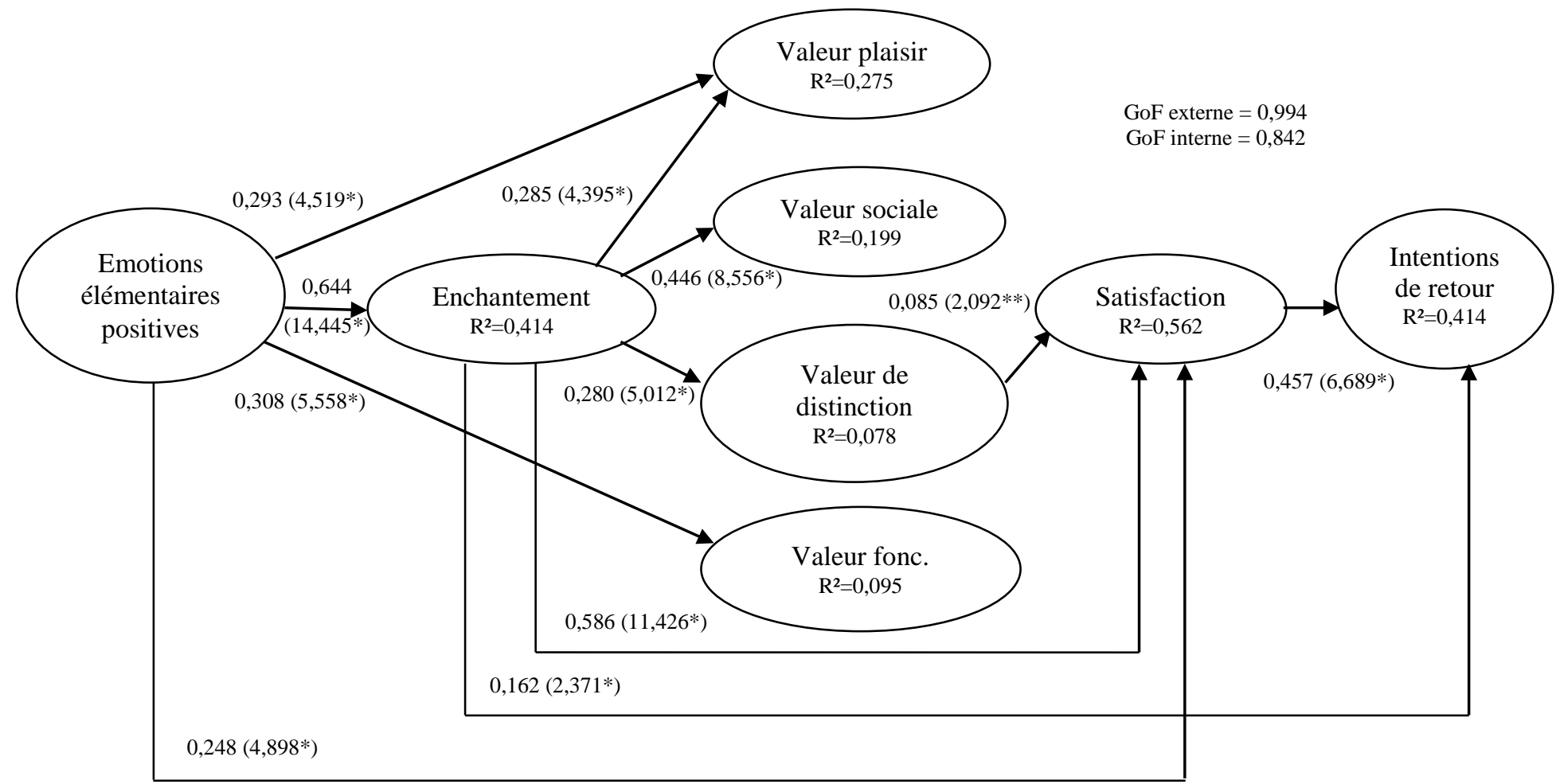

* Coefficient significatif. Les valeurs du test en $t$ de Student supérieures à |2,575| indiquent des paramètres significatifs au seuil de $1 \%$.

** Coefficient significatif. Les valeurs du test en $t$ de Student supérieures à $|1,96|$ indiquent des paramètres significatifs au seuil de $5 \%$.

*** Coefficient significatif. Les valeurs du test en $t$ de Student supérieures à $|1,645|$ indiquent des paramètres significatifs au seuil de $10 \%$. 
Figure 3 : Résultats du modèle d’équations structurelles - Arc 1950 (n=244)

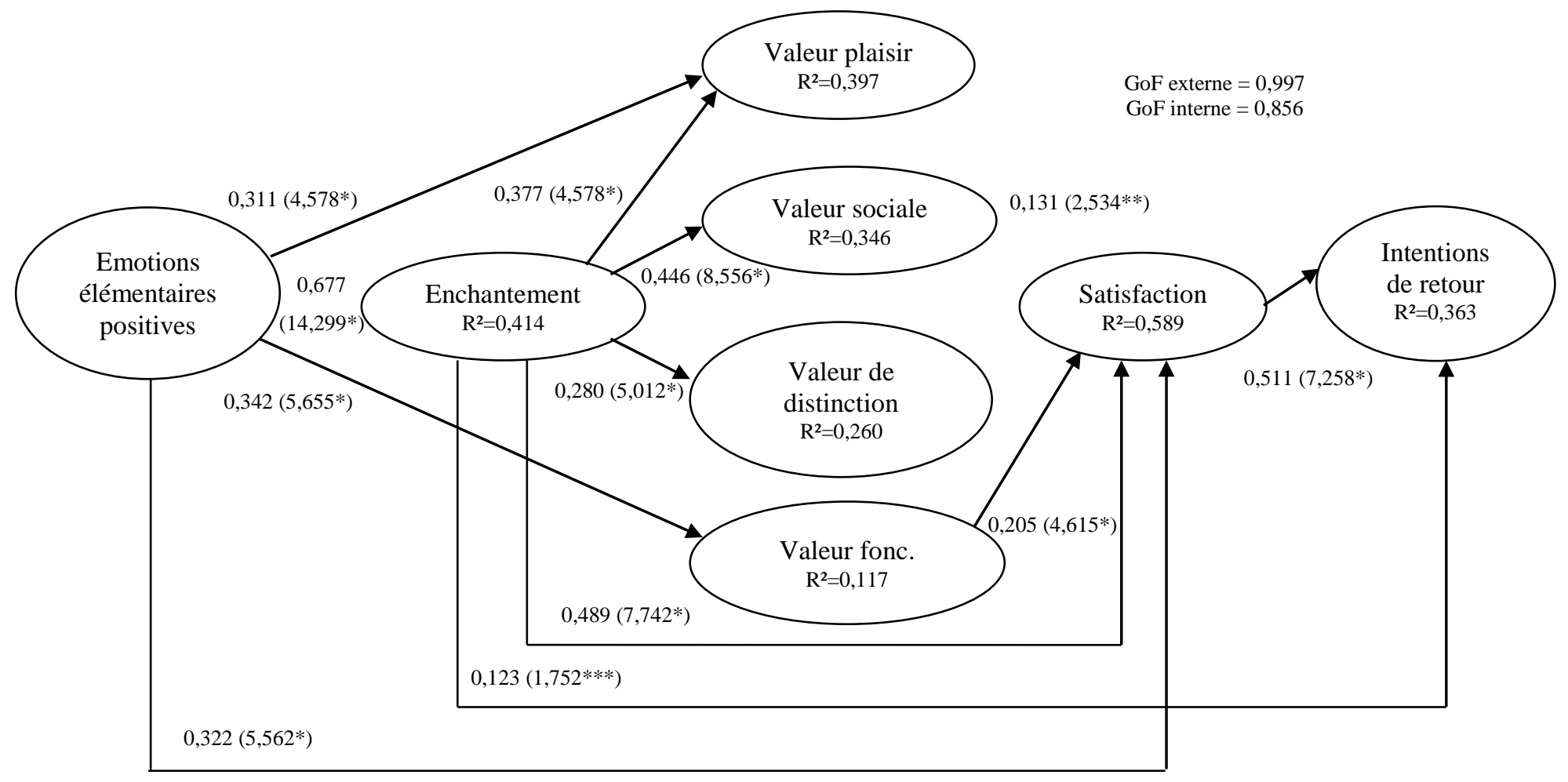

* Coefficient significatif. Les valeurs du test en $t$ de Student supérieures à $|2,575|$ indiquent des paramètres significatifs au seuil de 1 \%.

** Coefficient significatif. Les valeurs du test en $t$ de Student supérieures à $|1,96|$ indiquent des paramètres significatifs au seuil de $5 \%$.

*** Coefficient significatif. Les valeurs du test en $t$ de Student supérieures à $|1,645|$ indiquent des paramètres significatifs au seuil de $10 \%$. 
Encadré 1 : Présentation des deux villages de la station des Arcs étudiés

La station de sports d’hiver des Arcs (Savoie) est composée de quatre sites désignés par leur altitude approximative. Parmi ces quatre sites, Arc 1800 et Arc 1950 sont des stations qui proposent des offres pour des «vacanciers en quête d'expérience ». Les deux stations adoptent un positionnement expérientiel. Cependant, si Arc 1800 a été construite à partir des années 1970 pour répondre avant tout à la demande croissante de touristes skieurs, Arc 1950 (construite au début des années 2000) propose une expérience totale et spectaculaire matérialisée par sa très forte théâtralisation. En effet, si Arc 1950 est une station intégrée où le séjour des clients est facilité (parking sous-terrain avec accès direct aux résidences, skis aux pieds dans l'ensemble du village, commerces sélectionnés), l'enrichissement de « l'expérience 1950 » est avant tout lié à « son décor, ses façades aux couleurs chatoyantes, le charme de ses places ». La station décline une vision idéalisée des villages savoyards traditionnels et du «ski d'autrefois » jouant ainsi au travers de son décor sur la nostalgie, la magie et l'enchantement. Ainsi, Arc 1950 est « un lieu harmonieux où la douceur de vivre des villages d'antan se conjugue avec les facilités de la vie moderne " et où les touristes repartiront avec «d'inoubliables souvenirs ». Arc 1950 propose donc une offre d'expérience enrichie, conduisant ses responsables à la présenter comme «un site sans équivalent en Europe ». 